\begin{tabular}{|c|c|}
\hline Citation & $\begin{array}{l}\text { Kim S., Mewis J., Clasen C., Vermant J. (2013), } \\
\text { Superposition rheometry of a wormlike micellar fluid. } \\
\text { Rheologica Acta. nr.52, pp. } 727-740 .\end{array}$ \\
\hline Archived version & $\begin{array}{l}\text { Author manuscript: the content is identical to the content of the published } \\
\text { paper, but without the final typesetting by the publisher }\end{array}$ \\
\hline Published version & $\begin{array}{l}\text { The final publication is available at Springer via } \\
\text { http://dx.doi.org/10.1007/s00397-013-0718-2 }\end{array}$ \\
\hline Journal homepage & http://link.springer.com/journal/397 \\
\hline Author contact & your email christian.clasen@cit.kuleuven.be \\
\hline IR & url in Lirias https://lirias.kuleuven.be/handle/123456789/401237 \\
\hline
\end{tabular}

(article begins on next page) 


\title{
Superposition rheometry of a wormlike micellar fluid
}

\author{
Sunhyung Kim, Jan Mewis, Christian Clasen and Jan Vermant* \\ Department of Chemical Engineering, KU Leuven, \\ University of Leuven \\ W. de Croylaan 46, B-3001, Heverlee, Belgium
}

\section{Synopsis}

Oscillatory measurements are often used to explore the non-linear response of materials, with recently a strong focus on using large amplitude oscillatory experiments. However, the superposition of an oscillatory motion onto a steady state shear flow is a method where the kinematic history experienced by the sample is simpler. Such a superposed oscillation can be applied either orthogonal or parallel to the main flow direction. Both superposed deformation modes can now be achieved on rotational rheometers equipped with a force-rebalanced transducer, the orthogonal mode requiring a minor modification to the control loop of the normal force. In the present work the non-linear properties of a wormlike micellar solution (WLM) are studied. The results are compared with the predictions of the Giesekus model, which is chosen both for its capability to describe the WLM response, and for being one of the simplest continuum models that incorporate an anisotropic microstructure. From the fluid response in the homogeneous flow regime, a rate dependent relaxation time and a rate dependent plateau modulus can be derived. The latter provide insight into the structural anisotropy during flow at short length scales, which in this case is isotropic. Further analysis of the superposition moduli can be used to separate and quantify the effects of flow on the reptation and breaking of the chains. In the shear-banding regime, the orthogonal moduli show a weaker dependence on shear rate compared to the predictions of the Giesekus model, yet they remain sensitive to changes in the shear banded state.

Keywords: orthogonal superposition, parallel superposition, wormlike micellar solution, Giesekus model, non-linear rheology 


\section{Introduction}

The recent surge in interest in the use of large amplitude oscillatory shear (LAOS) measurements demonstrates that the quest for adequate measurement techniques to probe the non-linear response of complex fluids is still ongoing (Hyun et al. 2011). Measurements in the frequency domain are appealing as the effects of small (SAOS) and large (LAOS) deformations on the different relaxation modes can be probed. However, LAOS data are only scalar in nature and the deformation history in a LAOS experiment is complex. Superposition of a small strain oscillatory motion onto a steady or transient shear flow can provide a clearer insight into the effects of flow on the mechanisms underlying the non-linear response of rheological complex fluids. As schematically demonstrated in Fig. 1, the oscillatory motion can be imposed parallel ( $\dot{\gamma}_{\square}$, parallel superposition) or perpendicular $\left(\dot{\gamma}_{\perp}\right.$, orthogonal superposition,) to the direction of the steady or transient shear flow $(\dot{\gamma})$. Such superposition techniques have been used to study structural changes during flow in various systems, including polymeric fluids (Osaki et al. 1965; Booij 1966a; Tanner and Simmons 1967a; Simmons 1968; Mewis et al. 2001; Somma et al. 2007), colloidal suspensions (Mewis and Schoukens 1978; Zeegers et al. 1995; van der Vorst et al. 1998; Dhont and Wagner 2001; Mewis and Biebaut 2001; Mobuchon et al. 2009) and liquid crystalline polymers (Moldenaers and Mewis 1993; Walker et al. 2000).

Due to the tensorial nature of the rheological response, orthogonal and parallel moduli are not equivalent as demonstrated by both measurements and model calculations (Tanner and Simmons 1967b; Tanner 1968; Vermant et al. 1998). In parallel superposition measurements, the main and the superimposed shear fields are coupled, which complicates the interpretation of the results. To illustrate this complexity it suffices to point out that parallel superposition can result in phase angles exceeding $90^{\circ}$, and consequently in negative in-phase moduli at low frequencies where shear flow overwhelms the relaxation mode of the material (Booij 1966a, b). In contrast to this, orthogonal superposition has a much weaker coupling of the two flow fields and hence the corresponding moduli can be more directly related to the microstructure under flow. 
Orthogonal superposition has intrinsic advantages over parallel superposition because of the decoupling from the steady shear flow. Yet, it has received only limited attention due to the technical difficulties in establishing such orthogonal superposition flows, whereas parallel superposition is now readily available on commercial rheometers. The orthogonal superposition technique has been introduced first by Simmons (1966) and similar devices have been described by Mewis and Schoukens (1978) and by Zeegers et al. (1995). However, these instruments have a rather complex and delicate mechanical design and only cover a rather narrow viscosity range. Vermant et al. (1997) have described an orthogonal superposition technique based on a simple modification of the force rebalance transducer in a commercial rheometer. Mobuchon et al. (2009) used the same modification to perform oscillatory motions in different straining directions, by means of which the anisotropy of the linear viscoelastic moduli of a presheared material could be probed (2D-SAOS).

Superposition moduli should be described by suitable rheological constitutive models. Lacking a generally valid non-linear rheological constitutive equation, authors have used various models. Booij (1966a, 1966b) compared the occurrence of negative parallel moduli at low frequencies for some polymeric solutions with predictions from the Oldroyd model, and showed a qualitative agreement. Tanner and co-workers (Tanner 1968, Tanner and Simmons 1967a) pointed out more clearly that orthogonal and parallel moduli are not equivalent, using the Lodge model. Yamamoto (1971) proposed a strain rate dependent relaxation spectrum, which can be used to demonstrate that parallel moduli reflect the coupling between shear and superimposed flow, while orthogonal superposition moduli do not. These results are also consistent with the analyses of Bernstein (1972) with the KBK-Z model and of Macdonald (1973) with the Bird-Carreau model. De Cleyn and Mewis (1981) proposed a constitutive equation based on the concept of structural kinetics and used it to study the behavior during and after flow by means of orthogonal oscillatory flow. Wong and Isayev (1989) predicted the orthogonal moduli with the Leonov model, later corrected by Kwon and Leonov (1993). Vermant et al. (1998) used superposition moduli to evaluate rheological models including the Yamamoto, KBK-Z and Wagner model. Dhont and Nägele (1998) developed a model based on microscopic considerations to describe a viscoelastic response of superposition flows for suspension of spherical particles near the gas-liquid critical point. Using the same considerations, Dhont and Wagner (2001) predicted orthogonal and parallel moduli of colloidal system of attractive particles near their gas-liquid critical point and demonstrated a 
fundamental difference between the viscoelastic response to an orthogonal and parallel superposition flow.

In order to understand more clearly what is being measured in superposition rheometry, the present work focuses on the Giesekus model (Giesekus 1982a). It is known to describe the standard non-linear flow properties of wormlike micellar (WLM) solutions (Holz et al. 1999; Helgeson et al. 2009), and also represents one of the simplest continuum models which nevertheless incorporates an anisotropic microstructure, using the concept of deformationdependent tensorial mobility. WLM solutions contain flexible, wormlike surfactant micelles, and the rheological properties of the solutions can be readily tuned by e.g. concentration or temperature (Rehage and Hoffman, 1991). WLM solutions are sometimes called 'living' systems because they combine the well-known reptation dynamics of polymeric systems, with breakup and recombination of the chains (Cates 1987, 1990; Cates and Candau, 1990). Whereas WLM solutions are prone to flow instabilities known as shear banding (see e.g. Lerouge and Berret 2010, and references therein), the present study is limited to the regime where flow is homogeneous. In this regime, the segmental ordering induced by flow and the macroscopic rheological response have been qualitatively linked to the Giesekus model, for steady state flow conditions, using rheo-SANS and flow birefringence measurements (Liberatore et al. 2009) or LAOS (Rogers et al. 2013), but some discrepancies remain. In the present work superposition rheometry will be used to elucidate the link between the microstructural origin of the stresses and the success of the Giesekus model in describing them.

\section{Materials and Methods.}

\section{(1) Wormlike micelle solution}

The wormlike micelle solution investigated here is an aqueous solution containing $100 \mathrm{mM}$ cetylpyridinium chloride $(\mathrm{CPyCl}), 60 \mathrm{mM}$ sodium salicylate $(\mathrm{NaSal})$ and $100 \mathrm{mM} \mathrm{NaCl}$. This system is known to easily form elongated wormlike micelles, exhibiting a single relaxation time at equilibrium as it is in the fast breaking regime (Rehage and Hoffmann 1991). 


\section{(2) Rheological measurements}

Steady-state flow as well as parallel superposition and orthogonal superposition experiments were carried out using a ARES-G2 (TA instruments, Newcastle, Delaware) with a double wall Couette cell geometry. The first normal stress differences were measured with cone and plate geometry with a $2^{\circ}$ cone angle and a diameter of $50 \mathrm{~mm}$. All measurements were performed at $20.7^{\circ} \mathrm{C}$.

In orthogonal superposition measurements, an oscillatory motion is applied perpendicular to the main flow direction. This is achieved by modifying the ARES-G2 rheometer. In its normal operating mode the instrument uses a force-rebalanced transducer for the measurement of both torque and normal force. This rebalancing compensates the displacement of the transducer caused by the force in the sample. The axial rebalancing loop consists of three parts; a capacitive position measurement device, a motor to apply the rebalancing force and a control loop. The control loop for the normal force is modified here to drive an axial oscillatory motion, similar to a previous version of an orthogonal setup on an RMS 800 rheometer (Vermant et al. 1997). An oscillatory signal is generated by a frequency response generator and analyzer (FRA 1250, Solartron Instruments, Schlumberger) or alternatively using a high-accuracy multifunction data acquisition board, interfaced with LabVIEW (NI PCI-6281 and LabVIEW 8.5, National Instruments). This signal is introduced in the normal force control loop at the point where the signal generated by the position measurement sensor is fed back into the normal force control loop. The feedback loop forces the motor to follow the oscillatory signal. In this manner, a strain-controlled, axial oscillation of the transducer is generated. The magnitude of the axial force response is measured as an analog signal, using either the FRA or the combination of DAQ board with LabVIEW. To enable orthogonal superposition with the ARES-G2, TA Instruments has modified the normal force control board. The maximum frequency for the orthogonal oscillations is approximately $10 \mathrm{rad} / \mathrm{s}$, due to inertia effects. The lower frequency limit depends on the sample under consideration, due to the signal to noise ratio.

To create a uniform oscillatory deformation orthogonal to the main flow direction, a double wall Couette geometry has been used. A sinusoidal shear flow orthogonal to the main flows is obtained by moving a hollow bob axially, whereas the tangential steady shear flow is 
achieved by rotating the double-walled Couette cup. The axial motion may cause an annular pumping flow by displacing the fluid under the edge of the hollow bob. The pumping flow pushes liquid back up into the annular space between the bob and the inner and outer wall of the cup, thus distorting the flow field (Zeegers et al. 1995). To minimize the pumping effect during orthogonal superposition measurements, an opening is made in the inner wall of the cup that connects with a liquid reservoir in the center of the cup (Vermant et al. 1997). It has been verified that the small amplitude moduli measured with the orthogonal device at rest agree with the normal dynamic moduli obtained with rotational oscillatory shearing motions (Vermant et al. 1997).

\section{Predictions of the Giesekus model}

In the following we use the Giesekus model as a simple continuum model to generate predictions for the parallel and orthogonal moduli. These are validated against a WLM solution, whose non-linear rheological properties can be approximated by a single-mode Giesekus equation (Fischer and Rehage 1997; Holz et al. 1999; Helgeson et al. 2009; Gurnon and Wagner 2012).

\section{(1) Basic equation}

The Giesekus model was developed to model the non-linear rheological properties of polymers in solution but has been found to have wider applications, in particular for wormlike micelles. Giesekus extended the upper convected Maxwell model using a quadratic stress term and a mobility tensor to describe the non-linearity. The governing equation for the deviatoric, specific stress of the elastic component is given by (Giesekus 1982a)

$\mathbf{B} \cdot \boldsymbol{\sigma}+G \lambda \stackrel{\nabla}{\mathbf{C}}=0$

where $\mathbf{B}$ is the mobility tensor, $\boldsymbol{\sigma}$ the specific stress tensor and $\mathbf{C}$ the configuration tensor. $G$ and $\lambda$ are the shear modulus and relaxation time, respectively. Here $\nabla$ designates the upper convected time derivative: $\stackrel{\nabla}{\mathbf{C}}=\frac{\partial}{\partial t} \mathbf{C}+v(\nabla \cdot \mathbf{C})-\nabla v^{T} \cdot \mathbf{C}-\mathbf{C} \cdot \nabla v$. Giesekus introduced this equation to describe the anisotropic mechanical properties of rod-like aggregates under flow. For simplicity, Giesekus coupled the mobility tensor to the configuration tensor by 
introducing a dimensionless mobility parameter, $\alpha$, with values between zero and unity so that

$$
\mathbf{B}=\mathbf{I}+\alpha(\mathbf{C}-\mathbf{I})
$$

The specific stress tensor, $\boldsymbol{\sigma}$, and the configuration tensor, $\mathbf{C}$, are related by

$$
\boldsymbol{\sigma}=G(\mathbf{C}-\mathbf{I})
$$

Inserting Eqs. (2) and (3) into Eq. (1) gives

$$
\alpha(\mathbf{C}-\mathbf{I})^{2}+(\mathbf{C}-\mathbf{I})+\lambda \stackrel{\nabla}{\mathbf{C}}=0
$$

Combining Eqs. (3) and (4) gives a more familiar form (Giesekus 1982b):

$$
\boldsymbol{\sigma}+\lambda \stackrel{\nabla}{\boldsymbol{\sigma}}+\frac{\alpha}{G} \boldsymbol{\sigma} \cdot \boldsymbol{\sigma}=2 \lambda G \mathbf{D}
$$

The analytical solution for the case of steady state shear flow was described by Giesekus (Giesekus 1982a) and is given for reference in the appendix.

\section{(2) Analytical solution for orthogonal superposition flow}

From the Giesekus model and using the kinematics of orthogonal superposition to a shear flow, an analytical expression for the orthogonal moduli can be calculated, as shown in the appendix. The resulting $G_{\perp}^{\prime}$ and $G_{\perp}^{\prime \prime}$ are given by:

$$
\begin{aligned}
& G_{\perp}^{\prime}=G g_{\perp}^{\prime}=G \frac{A \chi^{6}+B \chi^{4}+C \chi^{2}}{\chi^{6}+D \chi^{4}+E \chi^{2}+F} \\
& G_{\perp}^{\prime \prime}=G g_{\perp}^{\prime \prime}+\eta_{S} \omega=G \frac{H \chi^{5}+I \chi^{3}+J \chi}{\chi^{6}+D \chi^{4}+E \chi^{2}+F}+\eta_{S} \omega
\end{aligned}
$$

where $\chi$ is the reduced frequency, $\chi=\lambda \omega$, and $\eta_{\mathrm{s}}$ is the effective medium viscosity. The coefficients indicated as capital letters are functions of the parameter $\alpha, \lambda$ and $G$ as well as the shear rate and given in the Appendix. Fig. 2 displays $G_{\perp}^{\prime}$ and $G_{\perp}^{\prime \prime}$ calculated from Eqs. (6) and (7), respectively, for different shear rates with values for the other parameters chosen as $\alpha=0.5, G=1 \mathrm{~Pa}, \lambda=1 \mathrm{~s}$ and $\eta_{\mathrm{s}}=0.001 \mathrm{~Pa}$ s. For $\mathrm{De}=0$ the orthogonal moduli indeed 
coincide with the Maxwellian moduli. This is illustrated in Fig. 2 where the non-linear $G_{\perp}^{\prime}$ and $G_{\perp}^{\prime \prime}$ are compared with the linear moduli for the extended Maxwell model given by

$$
\begin{aligned}
G^{\prime} & =\frac{G \chi^{2}}{1+\chi^{2}} \\
G^{\prime \prime} & =\frac{G \chi}{1+\chi^{2}}+\eta_{s} \omega
\end{aligned}
$$

For De $>1$, the superposition moduli start to deviate from Maxwellian behavior, as can be seen in Fig. 2. At a main shear rate of $10 \mathrm{~s}^{-1}, G_{\perp}^{\prime}$ and $G_{\perp}^{\prime \prime}$ can be compared with a Maxwell fluid of $\mathrm{G}=0.308 \mathrm{~Pa}$ (the observed modulus in Fig. 2), $\lambda=0.308 \mathrm{~s}$ (obtained from the low frequency limit of the loss modulus $\left.G_{\perp}^{\prime \prime} / \omega G\right)$ and $\eta_{\mathrm{s}}=0.001 \mathrm{~Pa}$ s. The deviation becomes more pronounced as De is increased, for example the frequency dependence of $G_{\perp}^{\prime}$ is even more steep than predicted by a single linear relaxation mode.

\section{(3) Analytical solution for parallel superposition flow}

In the case of parallel superposition, steady shear and oscillatory flow are coupled in this superposition flow, which complicates the analysis. An approximate analytical expression for the parallel moduli can be derived, as shown in the appendix, resulting in:

$$
\begin{aligned}
& G_{\square}^{\prime}=G g_{\square}^{\prime}=G \frac{K \chi^{6}+L \chi^{4}+M \chi^{2}}{\chi^{6}+N \chi^{4}+O \chi^{2}+P} \\
& G_{\square}^{\prime \prime}=G g_{\square}^{\prime \prime}+\eta_{S} \omega=G \frac{Q \chi^{5}+R \chi^{3}+S \chi}{\chi^{6}+N \chi^{4}+O \chi^{2}+P}+\eta_{S} \omega
\end{aligned}
$$

The shear rate dependent coefficients represented by capital letters are also given in the Appendix. Fig. 3 displays the parallel superposition moduli calculated from Eqs. (10) and (11) as a function of $\chi$, using $\alpha=0.5, G=1 \mathrm{~Pa}, \lambda=1 \mathrm{~s}$ and $\eta_{\mathrm{s}}=0.001 \mathrm{~Pa}$ s and for shear rates between 0 and $5 \mathrm{~s}^{-1}$. The approximate analytical expression for the moduli is compared to a full numerical solution using evolution equations for the configuration tensor (Eqs. (39), (40) and (42)) for a single shear rate $\dot{\gamma}=5 \mathrm{~s}^{-1}$. Fig. 3 shows good agreement between 
numerical and analytical values for the parallel moduli at low and high frequencies, and only minor deviations in the intermediate frequency range. The out-of-phase moduli for both parallel and orthogonal superposition are proportional to the frequency in the low frequency limit. In both cases the plateau values of the in-phase moduli decrease with increasing De. However, unlike the orthogonal moduli, the calculated $G_{\square}^{\prime}$ at low frequencies show the appearance of a region of negative values as the shear rate increases.

\section{Results}

\section{(1) Conventional characterization of micellar solution}

The linear viscoelastic and steady state properties of the WLM fluid are investigated first. The dynamic moduli of WLMs are usually dominated by two competing processes: reptation and reversible breaking-recombination of the micellar chains (Cates and Candau 1990). Therefore, the relaxation time of a WLM is determined by the ratio of the characteristic breakup time, $\lambda_{\text {break, }}$, and the reptation time, $\lambda_{\text {rep. }}$. If $\lambda_{\text {break }}<<\lambda_{\text {rep }}$, the stress relaxation exhibits an exponential decay with a single relaxation time and hence can be described by a singlemode Maxwell model as in Eqs. (8) and (9) (Rehage and Hoffmann 1991), at least at low frequencies. Fig. 4 shows that the dynamic moduli of the WLM system can indeed be described by a Maxwell element modified by adding a viscous element to describe the upturn in $\mathrm{G}^{\prime \prime}$ at high frequencies. By fitting the data with Eqs. (8) and (9), we obtain $G=37 \mathrm{~Pa}, \lambda=$ $1.5 \mathrm{~s}$ and $\eta_{\mathrm{s}}=0.036 \mathrm{~Pa} \mathrm{~s}$.

Fig. 5 shows the steady state viscosity curve measured with a double gap Couette geometry. It displays a shear thinning regime starting at $\dot{\gamma}_{1}$ and then a shear banding regime starting at $\dot{\gamma}_{2}$, where the shear stress displays a plateau (Spenley et al. 1993). This behavior is consistent with earlier observations on the same material at similar concentrations (Rehage and Hoffmann 1991; Spenley et al. 1993; Miller and Rothstein 2007; Miller et al. 2009; Kim et al. 2010), as well as on other WLMs (Yesilata et al. 2006; Helgeson et al. 2009). The shear thinning behavior of WLMs can be linked with the increased segmental orientation of the wormlike micelles, as clearly demonstrated by birefringence and flow-SANS experiments (Rehage and Hoffmann 1991; Liberatore et al. 2009). The shear thinning behavior of a WLM can be well described by the Giesekus model (Fischer and Rehage 1997; Holz et al. 1999; Yesilata et al. 2006; Liberatore et al. 2009). The solid lines in Fig. 5 are calculated using the 
Giesekus model, Eq. (26), with $G=37 \mathrm{~Pa}, \lambda=1.5 \mathrm{~s}$ and $\eta_{\mathrm{s}}=0.036 \mathrm{~Pa}$ s from Fig. 4 and $\alpha=$ 0.5 . In this case, the measured value of $\eta_{0}=54 \mathrm{~Pa} \mathrm{~s}$ agrees well with the calculated value ( $\eta_{0}=G \lambda=55.5 \mathrm{~Pa} \mathrm{~s}$ ), derived from the Maxwell fit of the moduli. The Giesekus model predicts the shear-thinning regime quite well for $\dot{\gamma}<\dot{\gamma}_{2}$. An extension of the Giesekus model with an empirical gradient diffusion term to capture also the shear banding regime has been presented by Helgeson et al. (2009). The present study is mainly focused on the shearthinning behavior. The quantitative analysis of the shear banded state lies beyond the scope of the current work.

\section{(2) Orthogonal superposition}

The orthogonal in-phase and out-of-phase moduli, $G_{\perp}^{\prime}$ and $G_{\perp}^{\prime \prime}$, are measured as a function of frequency for several shear rates up to $\dot{\gamma}=10 \mathrm{~s}^{-1}$. It has been verified that the applied orthogonal strain amplitude, 0.02, was within the linear response regime. Fig. 6 displays the orthogonal moduli up to a $\dot{\gamma}=1.5 \mathrm{~s}^{-1}$ with the last shear rate already within the shear banding regime. At the onset of the shear-thinning regime the orthogonal moduli start to decrease from the zero-shear values. The $G_{\perp}^{\prime}$ and $G_{\perp}^{\prime \prime}$ of the WLM solution are compared with the predictions of the Giesekus model, Eqs. (6) and (7), using the parameters which were obtained from the data for the normal dynamic moduli in Fig. 4 and the steady state shear flow in Fig. $5\left(\alpha=0.5, \lambda=1.5 \mathrm{~s}, G=37 \mathrm{~Pa}\right.$ and $\left.\eta_{\mathrm{s}}=0.036 \mathrm{~Pa} \mathrm{~s}\right)$. The agreement between experiment and Giesekus model for shear rates below the onset of shear banding is shown to be within experimental accuracy (Fig. 6), implying that the Giesekus model can indeed describe the rate-dependent superposition moduli of the WLM in quite some detail. The two dominant features in the data are the expected decrease of both moduli at low frequencies and the decrease of the plateau modulus with increasing shear rate, a feature quite different from typical results on polymer solutions (Vermant et al. 1998). Meanwhile, the high frequency values of $G_{\perp}^{\prime \prime}$ for both the Giesekus model and in the experiments do not change significantly with shear rate.

Fig. 7 displays the orthogonal moduli in the shear banding regime, for shear rates ranging from $\dot{\gamma}=1.5 \mathrm{~s}^{-1}$ to $\dot{\gamma}=10 \mathrm{~s}^{-1}$. For higher shear rates the results became erratic. The shear rates correspond to a significant fraction of the stress plateau in Fig. 5, however, the high 
shear band could not be accessed experimentally. In the shear banding regime, the orthogonal moduli of both $G_{\perp}^{\prime}$ and $G_{\perp}^{\prime \prime}$ still show the same trend as shown in the shear thinning regime in Fig. 6. The Giesekus model predicts, however, a more pronounced decrease. Interestingly, despite the observation that the overall stress is constant, the superposition moduli change with increasing shear rate. Unfortunately, the high shear rate band is outside of our experimental window, both in terms of its shear rate and relaxation time, making a quantitative analysis of the shear banding not possible. A direct comparison with parallel moduli is less evident in the shear banded state, as the different geometry may result in a different non-homogeneous flow profile.

\section{(3) Parallel superposition}

The parallel superposition moduli, $G_{\square}^{\prime}$ and $G_{\square}^{\prime \prime}$ were measured on the same system and over the same range of shear rates as for the orthogonal moduli. They are shown in Fig. 8 and are compared with the predictions of the Giesekus model, Eqs. (10) and (11), using the same parameters as used for orthogonal superposition. The model prediction is in good agreement with the experimental results up to $\dot{\gamma}=0.9 \mathrm{~s}^{-1}$, considerable deviations are observed at low frequencies at $\dot{\gamma}=1.5 \mathrm{~s}^{-1}$ which is, however, already in the shear banding regime.

The calculated negative values for $G_{\square}^{\prime}$ at low frequencies can be attributed to the coupling of the parallel moduli to the shear flow, as already demonstrated in earlier work (Booij 1966a, b). The simplest physical picture that rationalizes the low frequency behavior of the parallel moduli is based on Yamamoto's variable spectrum approach (Yamamoto 1971; Vermant et al. 1998), where the parallel modulus is found not only to be determined by the relaxation spectra itself but also by the derivative of these spectra with respect to the shear rate, as a result of the coupling of the two types of flow.

\section{Discussion}

\section{(1) Comparison of orthogonal and parallel moduli}

In Fig. 9, the measured orthogonal and parallel superposition moduli and predictions from the Giesekus model are compared at a single shear rate, $\dot{\gamma}=0.9 \mathrm{~s}^{-1}$. At low frequencies, $G_{\perp}^{\prime}$ exhibit a terminal behavior of $G_{\perp}^{\prime} \sim \omega^{2}$ whereas for $G_{\square}^{\prime}$ Giesekus predicts a limiting low frequency region with negative values in Eq. (10). At high frequencies, the values of the 
orthogonal and parallel moduli are identical. This holds in particular for the shear rate dependent plateau values of $G_{\perp}^{\prime}$ and $G_{\square}^{\prime}$ (Fig. 10a). This suggests that in the present case there is, at each shear rate, a critical frequency above which parallel moduli are not affected by the coupling with the steady shear flow. Such identical values for orthogonal and parallel moduli in the high frequency regime are not a universal feature. For other types of fluids, the relative magnitudes of $G_{\perp, \infty}^{\prime}$ and $G_{\square, \infty}^{\prime}$ enable one to evaluate the structural anisotropy of materials whenever the coupling between steady and parallel superposed shear flow can be neglected. This was the case for tests after cessation of flow on suspensions of layered silicate (Mobuchon et al. 2009). For liquid crystal polymers Mewis and coworkers (Walker et al. 2000) reported different magnitudes of parallel and orthogonal for the in-phase moduli over the whole frequency range which persisted even after the flow was arrested. Combining relevant rheological and rheo-optical results led to the conclusion that this difference between $G_{\perp, \infty}^{\prime}$ and $G_{\square, \infty}^{\prime}$ indeed arose from an anisotropic microstructure (Moldenaers and Mewis 1993). The experimental results on the WLM suggest isotropy at the (short) length scales corresponding to the high frequencies used here. Liberatore et al. (2009) investigated the shear-induced structure of (non-shear banding) WLMs at different length scales by means of combined flow-birefringence and flow-SANS experiments. These authors showed that only after substantial flow alignment of the overall WLM microstructure significant segmental alignment was observed. This is in agreement with the current observations. The regime of segmental orientation is obscured for the current system due to the occurrence of shear banding. The Giesekus model also predicts identical high frequency values, in agreement with the experiments. The high frequency limit of the orthogonal modulus $G_{\perp, \infty}^{\prime}$ is, according to Eq. (6), directly linked to the coefficient $A$ (see Appendix):

$G_{\perp, \infty}^{\prime} \equiv \lim _{\chi \rightarrow \infty} G_{\perp}^{\prime}=A \cdot G$

Similarly for the parallel modulus $G_{\square, \infty}^{\prime}$ in Eq. (10) the high frequency limit is

$$
G_{\square, \infty}^{\prime} \equiv \lim _{\chi \rightarrow \infty} G_{\square}^{\prime}=K \cdot G,
$$

with coefficient $K$ (see Appendix) identical to $A$. 


\section{(2) Plateau modulus}

In Fig. 10a, the identical values of $G_{\perp}^{\prime}$ and $G_{\square}^{\prime}$ at high frequencies (Figs. 6a and 8a), further referred to as $G_{\infty}^{\prime}$, exhibit a systematic decrease with increasing shear rate. The decrease can be linked to shear induced changes in the mesh size of the micellar network. According to the theory of rubber elasticity, the plateau modulus is related to the mesh size, $\xi$, by $G_{\infty}^{\prime}=k_{B} T / \xi^{3}$ ( Rubenstein and Colby 2003; Larson 2012). At rest this yields a value of $\xi$ $=48 \mathrm{~nm}$ which is of the same order of magnitude as those reported for other WLMs at a similar concentrations (Berret et al. 1993, Schubert et al. 2003). Under flow the values of $\xi$ in Fig. $10 \mathrm{~b}$ increase from $\xi=48 \mathrm{~nm}$ at $\dot{\gamma}=0 \mathrm{~s}^{-1}$ to $\xi=56.5 \mathrm{~nm}$ at $\dot{\gamma}=1.5 \mathrm{~s}^{-1}$.

From the parameters of the Giesekus model, as derived from the standard rheological measurements, the normal stress differences can be calculated. This requires either non-linear fitting of the rheological data (Fischer and Rehage 1997; Calin et al. 2010; Snijkers et al. 2011), or a direct calculation from LAOS data (Gurnon and Wagner 2012). Similarly to the LAOS experiments the values for the normal stress components can also be obtained directly, without complex fitting procedures, from the in-phase superposition moduli, e.g. for $N_{2}$ :

$G_{\infty}^{\prime}(\dot{\gamma})=G_{\infty}^{\prime}(\dot{\gamma}=0)-N_{2}$

In the Giesekus model the observable superposition moduli are directly linked to the normal stress differences. The predicted normal stress differences $\left(N_{1}\right.$ and $\left.N_{2}\right)$ according to the different procedures are shown in Fig. 11 and for $N_{l}$ they are compared to the experimentally measured values. Below the onset of shear banding the results are consistent.

\section{(3) Relaxation time}

The Giesekus model provides an effective shear rate dependent relaxation time $\bar{\lambda}=N_{1} /(2 \dot{\gamma} \sigma)$ that can directly be obtained from model parameters via Eq. (27). Results are shown in Fig. 12 and show a decrease of $\bar{\lambda}$ from the initial $\lambda$ with increasing rate. This effective relaxation time is experimentally accessible via the steady shear stress and first 
normal stress difference. However, the signal to noise ratio for $\mathrm{N}_{1}$ is not good for the WLM's and an accurate determination of $\bar{\lambda}$ in this manner is not possible. An alternative experimental measure can be obtained from the orthogonal superposition moduli. The crossover relaxation time of the orthogonal moduli $\bar{\lambda}_{\perp, \delta=\pi / 2}$, calculated from the Giesekus model, shows a good agreement with the effective relaxation time in Fig. 12. It can hence be used as an approximation of the effective relaxation time, $\bar{\lambda}$. Results of the experimentally obtained $\bar{\lambda}_{\perp, \delta=\pi / 2}$ are also given in Fig. 12 and are slightly below the theoretical ones, which can be traced to the slight discrepancy of the measured and calculated orthogonal loss moduli at higher frequencies (Fig. 6). As the shear rate is increased into the shear banding regime, the deviations from the basic form of the Giesekus model become obvious. For the parallel moduli the coupling effect with the steady shear flow makes the calculation of a relaxation time from the parallel superposition data mathematically ill-posed.

The crossover relaxation time is less well defined as the average or terminal relaxation time. In the current case it is, however, closer to the effective value as it originates from the higher frequency regime and is therefore less affected by the increasing deviation from a single mode Maxwell behavior at lower frequencies, as shown in Fig. 2. An average orthogonal relaxation time $\bar{\lambda}_{\perp, \omega \rightarrow 0}$ that is directly defined from the low frequency limits of the moduli

$$
\frac{\bar{\lambda}_{\perp}}{\lambda}=\lim _{\omega \rightarrow 0} \frac{G_{\perp}^{\prime}}{G_{\perp}^{\prime \prime} \omega}=\frac{C}{J}
$$

gives considerably lower values than the effective relation time in Fig. 12, an emanation of the intrinsic non-linearity as probed by superposition flows. Rogers et al. (2013) observed similar trends for the relaxation time as a function of Deborah number in LAOS experiments. However, as the moduli in the non-linear regime were fitted using a single-mode Maxwell model, this may underpredict the effective relaxation time.

According to the Giesekus model (Giesekus 1982a), the reduction of the rate-dependent relaxation time by the imposed shear flow is linked to a change in the configuration tensor. The latter can be characterized by the orientation angle, $\varphi$, between the first principle direction of the configuration tensor and the flow direction. Giesekus derived a simple 
relation between $\varphi$ and the rate-dependent effective relaxation time, which is given by (Giesekus 1982a):

$$
\cot \varphi=\bar{\lambda} \dot{\gamma}+\sqrt{1+\bar{\lambda}^{2} \dot{\gamma}^{2}}
$$

The orientation angles, calculated from the measured terminal relaxation times $\bar{\lambda}_{\perp, \delta=\pi / 2}$ (Fig. 13), show the expected gradual decrease with shear rate. The increase of the orientation and the decrease in relaxation time find their origin in the microstructural response of the WLM to flow. As previously mentioned, Cates (1987) suggested that the dynamics of WLM is dominated by the combination of the breakup-recombination motion and the reptation motion of micellar chains. In the fast breaking regime $\left(\lambda_{\text {break }}<<\lambda_{\text {rep }}\right)$, the WLM has a single relaxation time, in the absence of flow expressed by

$\lambda=\left(\lambda_{b r} \lambda_{r e p}\right)^{1 / 2}$

The extension of the Cates model to flowing conditions is based on the assumption that nonlinearities only affect the reptation motion, not the breakup of chains (Cates 1990, Cates and Candau 1990). This assumption was supported by comparing measured and calculated shear stresses for of WLM (Spenley et al. 1993). Based on the Cates model, the drop in relaxation time should be consistent with the changes in $\lambda_{\mathrm{br}}$ and $\lambda_{\text {rep }}$ as derived from the superposition moduli. The values of $\lambda_{\text {br }}$ can be obtained from the frequency $\omega_{\min }$ where $G$ " reaches its minimum: $\quad \lambda_{\mathrm{br}}=1 / \omega_{\min }$ (Turner and Cates 1991; Kern et al. 1994). Fig. 8 shows that $\omega_{\min }=$ $2.5 \mathrm{rad} / \mathrm{s}$, thus $\lambda_{\mathrm{br}}=0.04 \mathrm{~s}$ at $\dot{\gamma}=0 \mathrm{~s}^{-1}$. The values of $\lambda_{\mathrm{br}}$ turn out to be independent of the shear rate, thus confirming the assumption of Cates and coworkers. Although $\omega_{\min }$ could not measured in orthogonal superposition due to the mechanical limitations in frequency range, it is expected that similar values for $\omega_{\min }$ would be found as in parallel superposition because of their identical behavior at high frequencies, as previously discussed. The evolution of $\lambda_{\text {rep }}$ with shear rate is now calculated from $\lambda_{\text {br }}$ and $\bar{\lambda}_{\perp, \delta=\pi / 2} \quad$ (Fig. 12) using Eq. (17). The resulting values of $\lambda_{\text {rep }}$ (Fig. 14) decrease from $37.5 \mathrm{~s}$ at $\dot{\gamma}=0 \mathrm{~s}^{-1}$ to $16.7 \mathrm{~s}$ at $\dot{\gamma}=1.5 \mathrm{~s}^{-1}$, suggesting that the WLM chains are disentangling due to the shear flow. We note that $\lambda_{\text {break }}<<\lambda_{\text {rep }}$ in 
the range of shear rate under investigation, which is consistent with the orthogonal moduli exhibiting a single-mode behavior within the shear thinning regime.

\section{Conclusion}

In this study, the non-linear rheological properties of a WLM solution were explored by means of orthogonal and parallel superposition measurements. The Giesekus model has been selected as a simple constitutive model that allows a quantitative description of the shear thinning behavior of the WLM solutions up to the shear banding regime. For this model, analytical solutions for both orthogonal and parallel superposition moduli were obtained, which agree well with the experimental observations. The model calculations predict a linear relation between the orthogonal moduli and the normal stress differences, which can be obtained without the need for non-linear data fitting. This is confirmed by the experimental data. The superposition moduli can also be used to derive structural parameters. By varying the frequency, multiple length scales can be probed. An evaluation of the high frequency inphase moduli can be used to estimate the structural anisotropy on the segmental length scales, which was shown to be isotropic at the onset of the shear banding regime. The low frequency data show that the onset of the shear thinning response is associated with a larger scale anisotropy and orientation, in qualitative agreement with earlier SANS and birefringence results. Moreover, the assumption in the microstructural Cates model that the micellar breakup times are not affected by flow is proven to be correct.

\section{Acknowledgement}

Financial support from the ERC starting grant 203043 NANOFIB is gratefully acknowledged. Technical support of TA Instruments and financial support from EU FP7 (2011/2014) grant 262348 ESMI to modify the rheometer for orthogonal superposition measurements is also gratefully acknowledged. The assistance of Tom Verwijlen in the numerical calculations of the parallel moduli is acknowledged. 


\section{References}

Bernstein B (1972) A rheological relation between parallel and transverse superposed complex dynamic shear moduli. Rheol Acta 11: 210-215

Berret JF, Appell J, Porte G (1993) Linear rheology of entangled wormlike micelles. Langmuir 9: 2851-2854

Booij HC (1966a) Influence of superimposed steady shear flow on the dynamic properties of non-Newtonian fluids. Rheol Acta 5: 215-221

Booij HC (1966b) Influence of superimposed steady shear flow on the dynamic properties of non-Newtonian fluids. Rheol Acta 5: 222-227

Calin A, Wilhelm M, Balan C (2010) Determination of the non-linear parameter (mobility factor) of the Giesekus constitutive model using LAOS procedure. J Non-Newtonian Fluid Mech 165: 1564-1577

Cates ME (1987) Reptation of living polymers: dynamics of entangled polymers in the presence of reversible chain-scission reactions. Macromolecules 20: 2289-2296

Cates ME (1990) Nonlinear viscoelasticity of wormlike micelles (and other reversibly breakable polymers). J Phys Chem 94: 371-375

Cates ME, Candau SJ (1990) Statics and dynamics of worm-like surfactant micelles. J Phys-Condens Matter 2: 6869

De Cleyn G, Mewis J (1981) Constitutive equation for polymer liquids: application to shear flow. J Non-Newton Fluid Mech 9: 91-105

Dhont JKG, Nägele G (1998) Critical viscoelastic behavior of colloids. Phys Rev E 58: 7710-7732

Dhont JKG, Wagner NJ (2001) Superposition rheology. Phys Rev E 63: 021406

Fischer P, Rehage H (1997) Non-linear flow properties of viscoelastic surfactant solutions. Rheol Acta 36: 13-27

Giesekus H (1982a) A simple constitutive equation for polymer fluids based on the concept of deformation-dependent tensorial mobility. J Non-Newtonian Fluid Mech 11: 69-109

Giesekus H (1982b) A unified approach to a variety of constitutive models for polymer fluids based on the concept of configuration-dependent molecular mobility. Rheol Acta 21: 366-375 
Gurnon AK, Wagner NJ (2012) Large amplitude oscillatory shear (LAOS) measurements to obtain constitutive equation model parameters: Giesekus model of banding and nonbanding wormlike micelles. J Rheol 56: 333-351

Helgeson ME, Vasquez PA, Kaler EW, Wagner NJ (2009) Rheology and spatially resolved structure of cetyltrimethylammonium bromide wormlike micelles through the shear banding transition. J Rheol 53: 727-756

Holz T, Fischer P, Rehage H (1999) Shear relaxation in the nonlinear-viscoelastic regime of a Giesekus fluid. J Non-Newtonian Fluid Mech 88: 133-148

Hyun K, Wilhelm M, Klein CO, Cho KS, Nam JG, Ahn KH, Lee SJ, Ewoldt RH, McKinley GH (2011) A review of nonlinear oscillatory shear tests: Analysis and application of large amplitude oscillatory shear (LAOS). Prog Polym Sci 36: 1697-1753

Kern F, Lequeux F, Zana R, Candau SJ (1994) Dynamic Properties of Salt-Free Viscoelastic Micellar Solutions. Langmuir 10: 1714-1723

Kim NJ, Pipe CJ, Ahn KH, Lee SJ, McKinley GH (2010) Capillary breakup extensional rheometry of a wormlike micellar solution. Korea-Aust Rheol J 22: 31-41

Kwon Y, Leonov AI (1993) Remarks on orthogonal superposition of small amplitude oscillations on steady shear flow. Rheol Acta 32: 108-112

Larson RG (2012) The lengths of thread-like micelles inferred from rheology. J Rheol 56: 1363-1374

Lerouge S, Berret JF (2010) Shear-Induced Transitions and Instabilities in Surfactant Wormlike Micelles, Adv Polym Sci , vol 230. Springer Berlin Heidelberg, pp 1-71

Liberatore MW, Nettesheim F, Vasquez PA, Helgeson ME, Wagner NJ, Kaler EW, Cook LP, Porcar L, Hu YT (2009) Microstructure and shear rheology of entangled wormlike micelles in solution. J Rheol 53: 441-458

Macdonald IF (1973) Parallel superposition of simple shearing and small amplitude oscillatory motions. Trans Soc Rheol 17: 537-555

Mewis J, Biebaut G (2001) Shear thickening in steady and superposition flows effect of particle interaction forces. J Rheol 45: 799-813

Mewis J, Kaffashi B, Vermant J, Butera RJ (2001) Determining Relaxation Modes in Flowing Associative Polymers Using Superposition Flows. Macromolecules 34: 1376-1383 
Mewis J, Schoukens G (1978) Mechanical spectroscopy of colloidal dispersions. Faraday Discuss Chem Soc 65: 58-64

Miller E, Clasen C, Rothstein J (2009) The effect of step-stretch parameters on capillary breakup extensional rheology (CaBER) measurements. Rheol Acta 48: 625-639

Miller E, Rothstein JP (2007) Transient evolution of shear-banding wormlike micellar solutions. J Non-Newtonian Fluid Mech 143: 22-37

Mobuchon C, Carreau PJ, Heuzey MC, Reddy NK, Vermant J (2009) Anisotropy of nonaqueous layered silicate suspensions subjected to shear flow. J Rheol 53: 517-538

Moldenaers P, Mewis J (1993) On the nature of viscoelasticity in polymeric liquid crystals. J Rheol 37: 367-380

Osaki K, Tamura M, Kurata M, Kotaka T (1965) Complex Modulus of Concentrated Polymer Solutions in Steady Shear1. J Phys Chem 69: 4183-4191

Rehage H, Hoffmann H (1991) Viscoelastic surfactant solutions: model systems for rheological research. Mol Phys 74: 933-973

Rogers S, Kohlbrecher J, Lettinga MP (2012) The molecular origin of stress generation in worm-like micelles, using a rheo-SANS LAOS approach. Soft Matter 8: 7831-7839

Rubenstein M, Colby RH (2003) Polymer Physics. Oxford University Press, USA

Schubert BA, Kaler EW, Wagner NJ (2003) The Microstructure and Rheology of Mixed Cationic/Anionic Wormlike Micelles. Langmuir 19: 4079-4089

Simmons JM (1966) A servo-controlled rheometer for measurement of the dynamic modulus of viscoelastic liquids. J Sci Instrum 43: 887

Simmons JM (1968) Dynamic modulus of polyisobutylene solutions in superposed steady shear flow. Rheol Acta 7: 184-188

Snijkers F, D'Avino G, Maffettone PL, Greco F, Hulsen MA, Vermant J (2011) Effect of viscoelasticity on the rotation of a sphere in shear flow. J Non-Newtonian Fluid Mech 166: 363-372

Somma E, Valentino 0, Titomanlio G, Ianniruberto G (2007) Parallel superposition in entangled polydisperse polymer melts: Experiments and theory. J Rheol 51: 9871005

Spenley NA, Cates ME, McLeish TCB (1993) Nonlinear rheology of wormlike micelles. Phys Rev Lett 71: 939-942 
Tanner RI (1968) Comparative Studies of Some Simple Viscoelastic Theories. Trans Soc Rheol 12: 155

Tanner RI, Simmons JM (1967a) Combined simple and sinusoidal shearing in elastic liquids. Chem Eng Sci 22: 1803-1815

Tanner RI, Simmons JM (1967b) An instability in some rate-type viscoelastic constitutive equations. Chem Eng Sci 22: 1079-1082

Turner MS, Cates ME (1991) Linear viscoelasticity of living polymers: a quantitative probe of chemical relaxation times. Langmuir 7: 1590-1594

van der Vorst B, van den Ende D, Tekin NA, Mellema J (1998) Viscoelastic behavior of an ordering latex suspension in a steady shear flow. Phys Rev E 57: 3115-3122

Vermant J, Moldenaers P, Mewis J, Ellis M, Garritano R (1997) Orthogonal superposition measurements using a rheometer equipped with a force rebalanced transducer. Rev Sci Instrum 68: 4090-4096

Vermant J, Walker L, Moldenaers P, Mewis J (1998) Orthogonal versus parallel superposition measurements. J Non-Newtonian Fluid Mech 79: 173-189

Walker LM, Vermant J, Moldenaers P, Mewis J (2000) Orthogonal and parallel superposition measurements on lyotropic liquid crystalline polymers. Rheol Acta 39: $26-37$

Wong CM, Isayev AI (1989) Orthogonal superposition of small and large amplitude oscillations upon steady shear flow of polymer fluids. Rheol Acta 28: 176-189

Yamamoto M (1971) Rate-dependent relaxation spectra and their determination. Trans Soc Rheol 15: 331-344344

Yesilata B, Clasen C, McKinley GH (2006) Nonlinear shear and extensional flow dynamics of wormlike surfactant solutions. J Non-Newtonian Fluid Mech 133: 73-90

Zeegers J, Ende D, Blom C, Altena EG, Beukema GJ, Mellema J (1995) A sensitive dynamic viscometer for measuring the complex shear modulus in a steady shear flow using the method of orthogonal superposition. Rheol Acta 34: 606-621 


\section{Appendix.}

\section{Giesekus model predictions}

1. Results of the Giesekus model for steady shear flow conditions

The case of steady state shear flow was described by Giesekus (Giesekus 1982a). For shear flow with a velocity gradient tensor

$$
\nabla v^{T}=\left(\begin{array}{ccc}
0 & \dot{\gamma} & 0 \\
0 & 0 & 0 \\
0 & 0 & 0
\end{array}\right)
$$

and a symmetric configuration tensor with $C_{13}=C_{23}=0$, Eq. (4) results in four independent equations:

$$
\begin{array}{lr}
\alpha\left(C_{11}^{2}+C_{12}^{2}\right)+(1-2 \alpha) C_{11}-(1-\alpha)-2 \lambda \dot{\gamma} C_{12} & =0 \\
\alpha\left(C_{12}^{2}+C_{22}^{2}\right)+(1-2 \alpha) C_{22}-(1-\alpha) & =0 \\
\alpha C_{33}^{2}+(1-2 \alpha) C_{33}-(1-\alpha) & =0 \\
\alpha\left(C_{11}+C_{22}\right) C_{12}+(1-2 \alpha) C_{12} & -\lambda \dot{\gamma} C_{22}=0
\end{array}
$$

Eq. (21) is not coupled with the other equations and gives $C_{33}=1$ as the only positive solution. For the sake of simplicity, the components of the configuration tensor can be written as:

$$
C_{11}-C_{22}=N_{1} / G, C_{22}-C_{33}=N_{2} / G, C_{12}=\sigma / G
$$

with the first and the second normal stress differences $N_{1}$ and $N_{2}$ and the specific shear stress $\sigma$. Solving Eqs. (19) - (22) Giesekus obtained (Giesekus 1982a) :

$$
\begin{aligned}
& N_{2}=G \frac{1-\Lambda}{1+(1-2 \alpha) \Lambda} \\
& N_{1}=\frac{2 N_{2}\left(G-\alpha N_{2}\right)}{\alpha\left(G-N_{2}\right)}
\end{aligned}
$$


and for the total shear stress

$\sigma_{\text {tot }}=\sigma+\eta_{s} \dot{\gamma}=\frac{\left(G-N_{2}\right)^{2}}{G+(1-2 \alpha) N_{2}} D e+\eta_{s} \dot{\gamma}$

where $\mathrm{De}=\lambda \dot{\gamma}, \eta_{\mathrm{s}}$ is an effective medium viscosity and $\Lambda$ is an effective relaxation time $\bar{\lambda}=N_{1} /(2 \dot{\gamma} \sigma)$ reduced by the relaxation time $\lambda$

$\Lambda=\frac{\bar{\lambda}}{\lambda}=\frac{N_{1}}{2 \mathrm{De} \cdot \sigma}$

for which the rate-dependency is expressed by

$\Lambda^{2}=\frac{1}{8 \alpha(1-\alpha) \mathrm{De}^{2}}\left[\sqrt{1+16 \alpha(1-\alpha) \mathrm{De}^{2}}-1\right]$

2. Orthogonal moduli

In the case of orthogonal superposition of sinusoidal oscillations on steady shear flow, the velocity gradient tensor (as shown in Fig. 1) takes on the form

$\nabla v=\left(\begin{array}{ccc}0 & 0 & 0 \\ \dot{\gamma} & 0 & \dot{\gamma}_{\perp} \\ 0 & 0 & 0\end{array}\right)$

where $\dot{\gamma}_{\perp}=\gamma_{0, \perp} \omega \cos \omega t$. Here, $\gamma_{0, \perp}$ and $\omega$ are the strain amplitude and the angular frequency of the superimposed motion. The components $\mathrm{C}_{13 \perp}$ and $\mathrm{C}_{23 \perp}$ of the configuration tensor for orthogonal superposition can be expressed as 
Combined with the velocity gradient tensor, Eq.(29), and a symmetric configuration tensor, Eq. (4) leads to six independent differential equations:

$$
\begin{array}{ll}
\alpha\left(C_{11 \perp}^{2}+C_{12 \perp}^{2}+C_{13 \perp}^{2}\right)+(1-2 \alpha) C_{11 \perp}-(1-\alpha)-2 \lambda \dot{\gamma} C_{12 \perp} & =0 \\
\alpha\left(C_{12 \perp}^{2}+C_{22 \perp}^{2}+C_{23 \perp}^{2}\right)+(1-2 \alpha) C_{22 \perp}-(1-\alpha) & =0 \\
\alpha\left(C_{13 \perp}^{2}+C_{23 \perp}^{2}+C_{33 \perp}^{2}\right)+(1-2 \alpha) C_{33 \perp}-(1-\alpha)-2 \lambda \dot{\gamma}_{\perp} C_{23 \perp} \quad=0 & =0 \\
\alpha\left(C_{11 \perp} C_{12 \perp}+C_{12 \perp} C_{22 \perp}+C_{13 \perp} C_{23 \perp}\right)+(1-2 \alpha) C_{12 \perp}-\lambda \dot{\gamma} C_{22 \perp} & =0 \\
\alpha\left(C_{11 \perp} C_{13 \perp}+C_{12 \perp} C_{23 \perp}+C_{13 \perp} C_{33 \perp}\right)+(1-2 \alpha) C_{13 \perp}+\lambda \frac{\partial C_{13 \perp}}{\partial t}-\lambda\left(\dot{\gamma} C_{23 \perp}+\dot{\gamma}_{\perp} C_{12 \perp}\right)=0 \\
\alpha\left(C_{12 \perp} C_{13 \perp}+C_{22 \perp} C_{23 \perp}+C_{23 \perp} C_{33 \perp}\right)+(1-2 \alpha) C_{23 \perp}+\lambda \frac{\partial C_{23 \perp}}{\partial t}-\lambda \dot{\gamma}_{\perp} C_{22 \perp}
\end{array}
$$

As long as the superposition flow is only a small perturbation, $\mathrm{C}_{13 \perp}$ and $\mathrm{C}_{23 \perp}$ are much smaller than the other components $\mathrm{C}_{11 \perp}, \mathrm{C}_{22 \perp}, \mathrm{C}_{33 \perp}$ and $\mathrm{C}_{12 \perp}$ and hence the corresponding stresses are not coupled to the components of the superimposed flow. Ignoring $\mathrm{C}_{13 \perp}$ and $\mathrm{C}_{23 \perp}$ reduces Eqs. (31) - (34) to Eqs. (19) - (22) and $\mathrm{C}_{11 \perp}, \mathrm{C}_{22 \perp}, \mathrm{C}_{33 \perp}$ and $\mathrm{C}_{12 \perp}$ can be described by Eqs. (23) - (28). $\mathrm{C}_{13 \perp}$ and $\mathrm{C}_{23 \perp}$ can be obtained by inserting Eq. (30) into the Eqs. (35) and (36) and evaluating their coefficients. From this, we finally obtain the orthogonal moduli $G_{\perp}^{\prime}$ and $G_{\perp}^{\prime \prime}$ as:

$$
\begin{aligned}
& G_{\perp}^{\prime}=G g_{\perp}^{\prime}=G \frac{A \chi^{6}+B \chi^{4}+C \chi^{2}}{\chi^{6}+D \chi^{4}+E \chi^{2}+F} \\
& G_{\perp}^{\prime \prime}=G g_{\perp}^{\prime \prime}+\eta_{S} \omega=G \frac{H \chi^{5}+I \chi^{3}+J \chi}{\chi^{6}+D \chi^{4}+E \chi^{2}+F}+\eta_{S} \omega
\end{aligned}
$$

where $\chi$ is the reduced frequency, $\chi=\lambda \omega$, and $\eta_{\mathrm{s}}$ is the effective medium viscosity. The coefficients indicated as capital letters are functions of the shear rate 


$$
\begin{aligned}
& A=1-n_{2} \\
& B=\left(1-n_{2}\right)\left(2 a^{2}-b\right)-\alpha(\sigma / G)^{2}\left(2+\alpha n_{1}-2 \alpha n_{2}\right) \\
& C=\left(1-n_{2}\right)\left(a^{4}-a^{2} b\right)-\alpha(\sigma / G)^{2}\left\{a^{3}+\left(1-\alpha n_{2}\right) a^{2}\right\} \\
& D=\left(1-\alpha n_{2}\right)^{2}+2\left(a^{2}-b\right) \\
& E=2\left(1-\alpha n_{2}\right)\left\{\left(1-\alpha n_{2}\right) a^{2}+a b\right\}+\left(a^{2}-b\right)^{2} \\
& F=\left\{\left(1-\alpha n_{2}\right) a^{2}+a b\right\}^{2} \\
& H=\left(1-n_{2}\right)\left(1-\alpha n_{2}\right)+\alpha(\sigma / G)^{2} \\
& I=\left(1-n_{2}\right)\left\{2\left(1-\alpha n_{2}\right) a^{2}+a b\right\}-\alpha(\sigma / G)^{2}\left\{\left(1-\alpha n_{2}\right) a-a^{2}+b\right\} \\
& J=\left(1-n_{2}\right)\left\{\left(1-\alpha n_{2}\right) a^{4}+a^{3} b\right\}-\alpha(\sigma / G)^{2}\left\{\left(1-\alpha n_{2}\right) a^{3}+a^{2} b\right\}
\end{aligned}
$$

with

$a=1+\alpha n_{1}-\alpha n_{2}$

$b=\alpha(\sigma / G)\{\mathrm{De}-\alpha(\sigma / G)\}$

where $n_{1}$ and $n_{2}$ are reduced first and second normal stress difference, respectively, that can be obtained from $n_{1}=N_{1} / G$ and $n_{2}=N_{2} / G$, and where $N_{1}, N_{2}$ and $\sigma$ are given in Eqs. (24) - (26). 


\section{Parallel moduli}

In the case of parallel superposition, the kinematics are expressed by

$$
\nabla v=\left(\begin{array}{ccc}
0 & 0 & 0 \\
\dot{\gamma}+\dot{\gamma}_{\square} & 0 & 0 \\
0 & 0 & 0
\end{array}\right)
$$

where $\dot{\gamma}_{\square}=\gamma_{0,} \omega \cos \omega t$. Here, $\gamma_{0, \square}$ and $\omega$ are strain amplitude and angular frequency, respectively. Steady shear and oscillatory flow are coupled in this superposition flow, which complicates the interpretation of parallel moduli in terms of the microstructure. The individual components of the configuration tensor for parallel superposition can be expressed as:

$$
\begin{aligned}
& C_{1 \square}=C_{11}+\gamma_{0, \square} l_{\square}^{\prime} \sin \omega t+\gamma_{0, l_{\square}} l_{\square}^{\prime \prime} \cos \omega t \\
& C_{22 \square}=C_{22}+\gamma_{0, \square} m_{\square}^{\prime} \sin \omega t+\gamma_{0, \square} m_{\square}^{\prime \prime} \cos \omega t \\
& C_{33 \square}=C_{33}+\gamma_{0, \square} o_{\square}^{\prime} \sin \omega t+\gamma_{0, \square} o_{\square}^{\prime \prime} \cos \omega t \\
& C_{12 \square}=C_{12}+\gamma_{0, \square} g_{\square}^{\prime} \sin \omega t+\gamma_{0, \Omega} g_{\square}^{\prime \prime} \cos \omega t
\end{aligned}
$$

where $C_{11}, C_{22}, C_{33}$ and $C_{12}$ are identical to the results for steady shear flow without superposition, Eqs. (23) - (28). Eq. (4) now results in the following four independent differential equations:

$$
\begin{array}{ll}
\alpha\left(C_{11 \square}^{2}+C_{12 \square}^{2}\right)+(1-2 \alpha) C_{11 \square}-(1-\alpha)+\lambda \frac{\partial C_{11 \square}}{\partial t}-2 \lambda\left(\dot{\gamma}+\dot{\gamma}_{\square}\right) C_{12 \square}=0 \\
\alpha\left(C_{12 \square}^{2}+C_{22 \square}^{2}\right)+(1-2 \alpha) C_{22 \square}-(1-\alpha)+\lambda \frac{\partial C_{22 \square}}{\partial t} & =0 \\
\alpha C_{33 \square}^{2}+(1-2 \alpha) C_{33 \square}-(1-\alpha)+\lambda \frac{\partial C_{33 \square}}{\partial t} & =0 \\
\alpha\left(C_{11 \square} C_{12 \square}+C_{12 \square} C_{22 \square}\right)+(1-2 \alpha) C_{12 \square}+\lambda \frac{\partial C_{12 \square}}{\partial t}-\lambda\left(\dot{\gamma}+\dot{\gamma}_{\square}\right) C_{22 \square} & =0
\end{array}
$$


Here, Eq. (41) is not coupled with the other equations and gives $C_{33}||=1$ as the only admissible solution. The expressions about $\mathrm{C}_{11 \mid}, \mathrm{C}_{22}||$ and $\mathrm{C}_{12}||$ are obtained by inserting Eq. (38) into Eqs. (39), (40) and (42) and evaluating their coefficients. When higher orders of the sine and cosine functions are neglected, an approximated analytical expression for the parallel moduli, $G_{\square}^{\prime}$ and $G_{\square}^{\prime \prime}$ can be obtained from $\mathrm{C}_{12||}$ in Eq. (38):

$$
\begin{aligned}
& G_{\square}{ }^{\prime}=G g_{\square}{ }^{\prime}=G \frac{K \chi^{6}+L \chi^{4}+M \chi^{2}}{\chi^{6}+N \chi^{4}+O \chi^{2}+P} \\
& G_{\square}{ }^{\prime \prime}=G g_{\square}{ }^{\prime \prime}+\eta_{S} \omega=G \frac{Q \chi^{5}+R \chi^{3}+S \chi}{\chi^{6}+N \chi^{4}+O \chi^{2}+P}+\eta_{S} \omega
\end{aligned}
$$

where

$$
\begin{aligned}
& \chi=\lambda \omega \\
& K=1-n_{2} \\
& L=c^{2} h+h e^{2}-2(c+f) \alpha(\sigma / G)^{2}+4 d h \alpha(\sigma / G) \\
& M=c^{2} e^{2} h+4(e-c) d \alpha^{2}(\sigma / G)^{3}-2(c+f) e^{2} \alpha(\sigma / G)^{2}+2\left(c^{2}+e^{2}\right) d h \alpha(\sigma / G) \\
& N=c^{2}+e^{2}+f^{2}+4 d \alpha(\sigma / G) \\
& O=c^{2} f^{2}+c^{2} e^{2}+e^{2} f^{2}+4\left(c^{2}-e f\right) d \alpha(\sigma / G) \\
& P=c^{2} e^{2} f^{2}+4\left(c^{2}-e^{2}\right) d^{2} \alpha^{2}(\sigma / G)^{2}-4 c^{2} d e f \alpha(\sigma / G) \\
& Q=f h+2 \alpha(\sigma / G)^{2} \\
& R=c^{2} f h+e^{2} f h+2\left(e^{2}-c f\right) \alpha(\sigma / G)^{2}+2(c-e) d h \alpha(\sigma / G) \\
& S=c^{2} e^{2} f h+4(c-e) d e \alpha^{2}(\sigma / G)^{3}-2 c e^{2} f \alpha(\sigma / G)^{2}+2(e-c) c d e h \alpha(\sigma / G)
\end{aligned}
$$

with

$$
\begin{aligned}
& c=1+2 \alpha\left(n_{1}-n_{2}\right) \\
& d=\alpha(\sigma / G)-\mathrm{De} \\
& e=1-2 \alpha n_{2} \\
& f=1+\alpha\left(n_{1}-2 n_{2}\right) \\
& h=1-n_{2}
\end{aligned}
$$




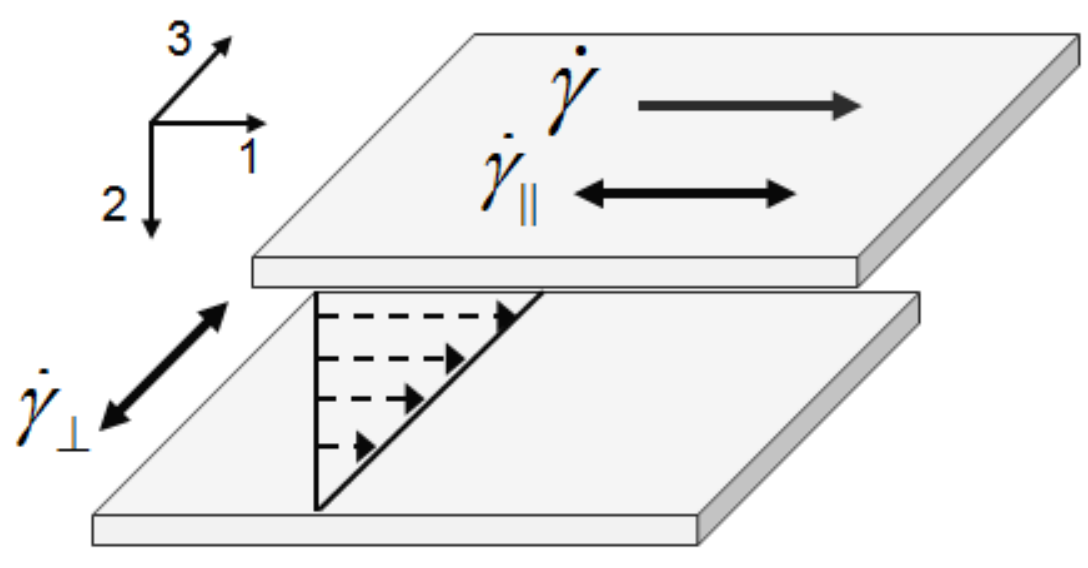

Fig. 1 Superposition flows: Orthogonal superposition is achieved by superposing the oscillatory flow in the vorticity direction $\left(\dot{\gamma}_{\perp}\right)$ onto the shear flow $(\dot{\gamma})$. Parallel superposition by oscillatory flow in the flow direction $\left(\dot{\gamma}_{\square}\right)$ onto the shear flow $(\dot{\gamma})$. 

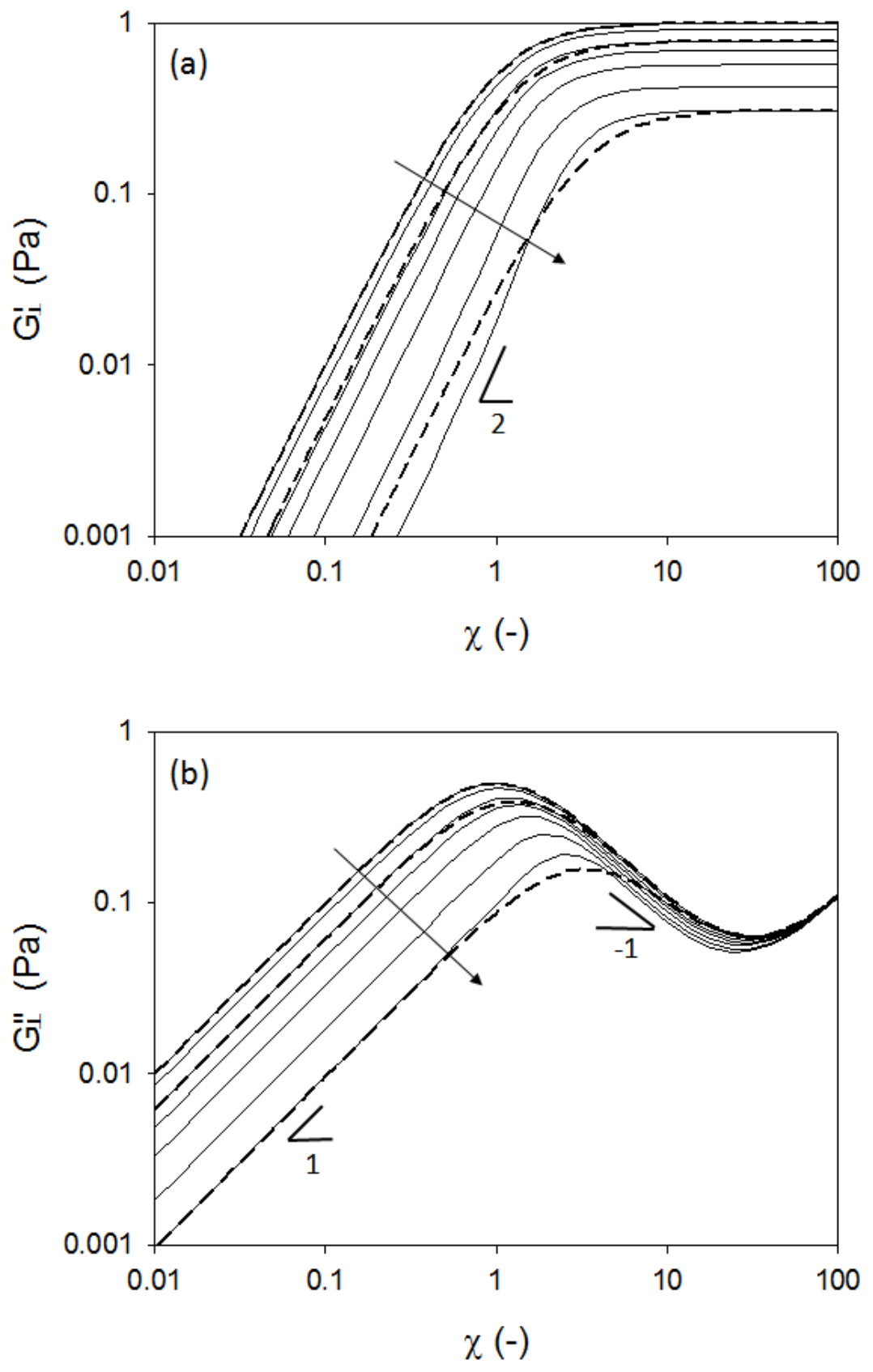

Fig. 2 Orthogonal moduli as a function of the reduced frequency $\chi . G_{\perp}^{\prime}$ (a) and $G_{\perp}^{\prime \prime}$ (b) calculated from the Giesekus model (solid lines), Eqs. (6) and (7), with $G=1 \mathrm{~Pa}, \lambda=1 \mathrm{~s}, \alpha=$ 0.5 and $\eta_{\mathrm{s}}=0.001 \mathrm{~Pa} \mathrm{~s}$. The shear rates for the different curves correspond to $\mathrm{De}=0,0.45,1$, 1.5. 2.5, 5 and 10 (from left to right as indicated by arrows). Predictions for the extended Maxwell model are shown, calculated using $G=1,0.786$ and $0.308 \mathrm{~Pa}, \lambda=1,0.786$ and $0.308 \mathrm{~s}$ and $\eta_{\mathrm{s}}=0.001 \mathrm{~Pa} . \mathrm{s}$, to illustrate the inherent difference between a linear viscoelastic and a superposition modulus as De increases. 

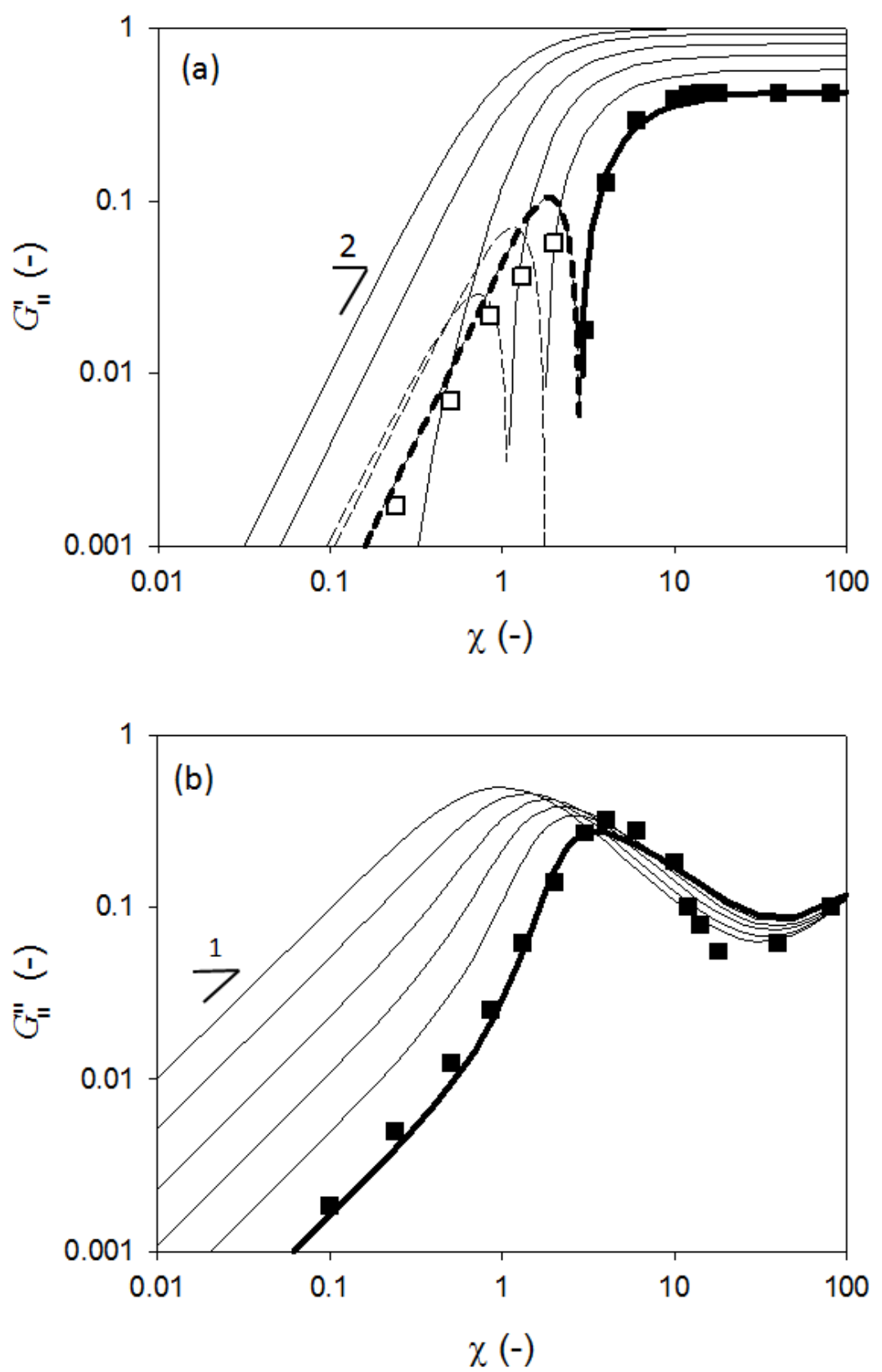

Fig. 3 Parallel moduli, $G_{\square}^{\prime}\left(\right.$ a) and $G_{\square}^{\prime \prime}$ (b) calculated from the Giesekus model as a function of reduced frequency, $\chi$. Solid lines are calculated from Eqs. (10) and (11) with same model parameters as in Fig. $2 ; \dot{\gamma}=0,0.45,0.9,1.5 .2 .5$ and $5 \mathrm{~s}^{-1}$ (left to right). Dashed lines in (a): the absolute value of the negative moduli at $\dot{\gamma}=1.5$. 2.5 and $5 \mathrm{~s}^{-1}$. The moduli at $\dot{\gamma}=5 \mathrm{~s}^{-1}$ are indicated thick lines. Symbols are the result of numerical calculation of Eqs. (39), (40) and (42) at $\dot{\gamma}=5 \mathrm{~s}^{-1}$. Open symbols: the absolute values of the negative moduli, close symbols: positive values. 


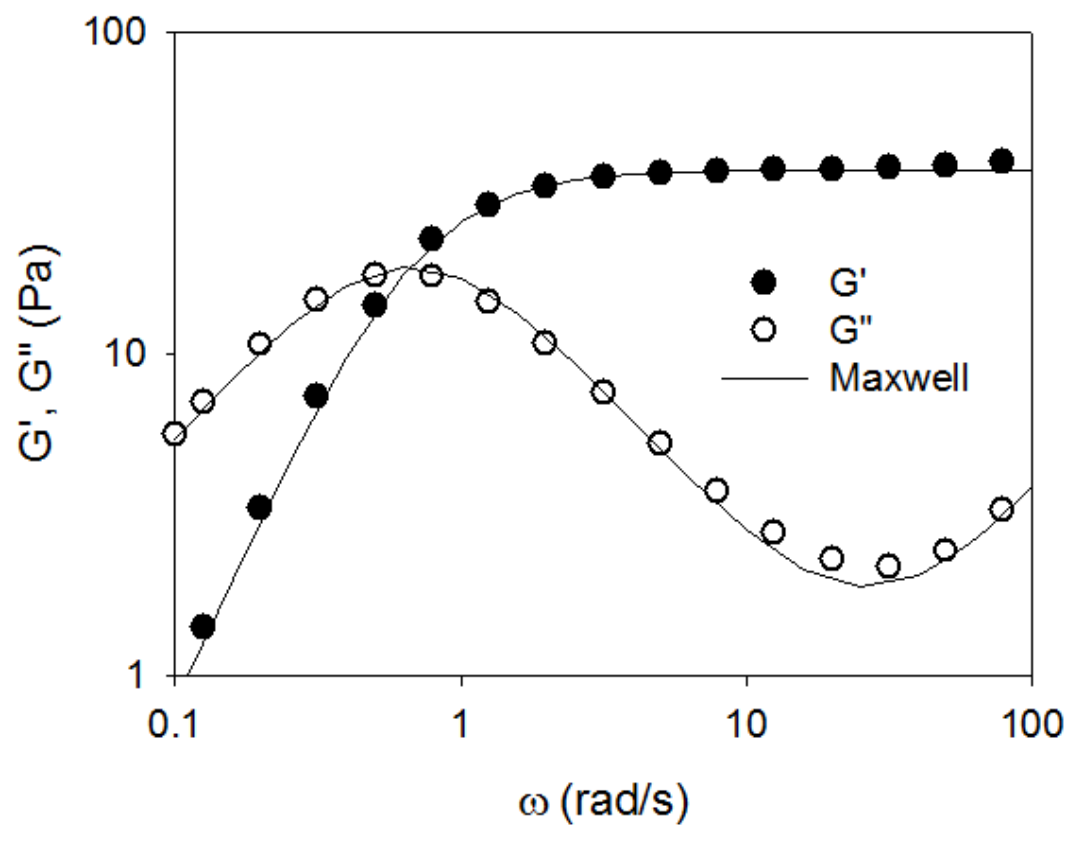

Fig. 4 Linear viscoelastic moduli of the WLM. Experimental values (symbols) and calculated values (solid lines) obtained by a fit of the extended Maxwell model, Eqs. (8) and (9), $G_{\infty}^{\prime}=37 \mathrm{~Pa}$ and $\lambda=1.5 \mathrm{~s}$, and $\eta_{\mathrm{s}}=0.036 \mathrm{~Pa} \mathrm{~s}$. 


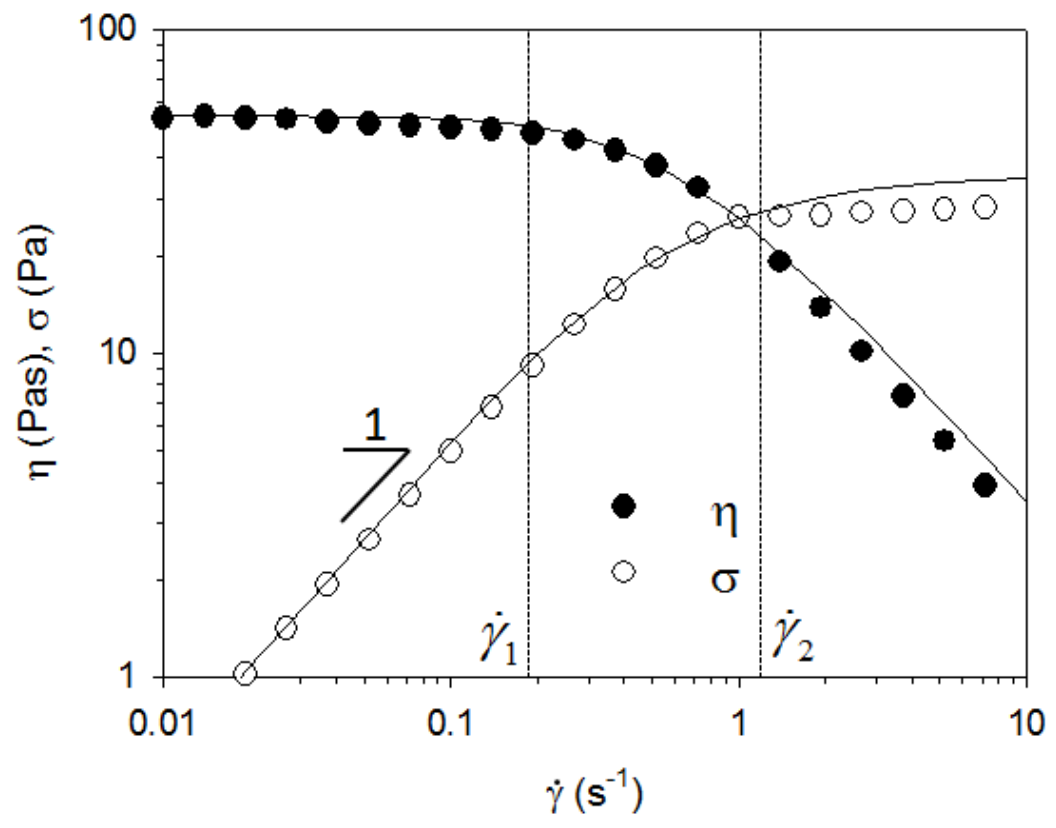

Fig. 5 Steady shear viscosity of the wormlike micelle solution. Symbols are measurements and solid lines are fit with the Giesekus model Eq. (26) with $G=37 \mathrm{~Pa}, \lambda=1.5 \mathrm{~s}, \eta_{\mathrm{s}}=0.036$ Pa s and $\alpha=0.5 . \quad \dot{\gamma}_{1}$ delimits the shear thinning regime, whereas $\dot{\gamma}_{2}$ indicates the shear rate at which the shear banding regime starts. 

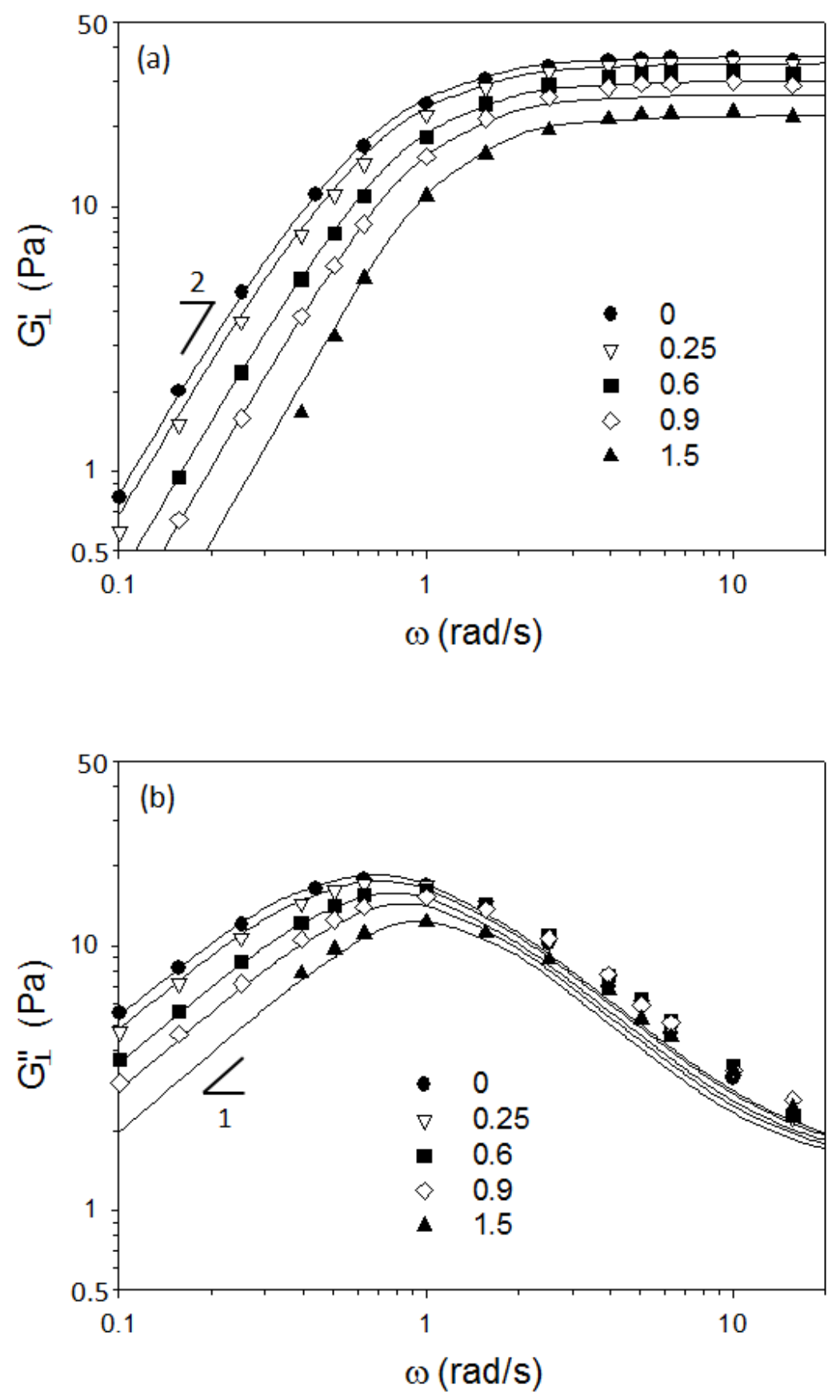

Fig. 6 Orthogonal superposition moduli, (a) $G_{\perp}^{\prime}$ and (b) $G_{\perp}^{\prime \prime}$ : experimental values (symbols), and calculated values (lines) from the Giesekus model, Eqs. (6) and (7) with model parameters same as used in Fig. 5. 

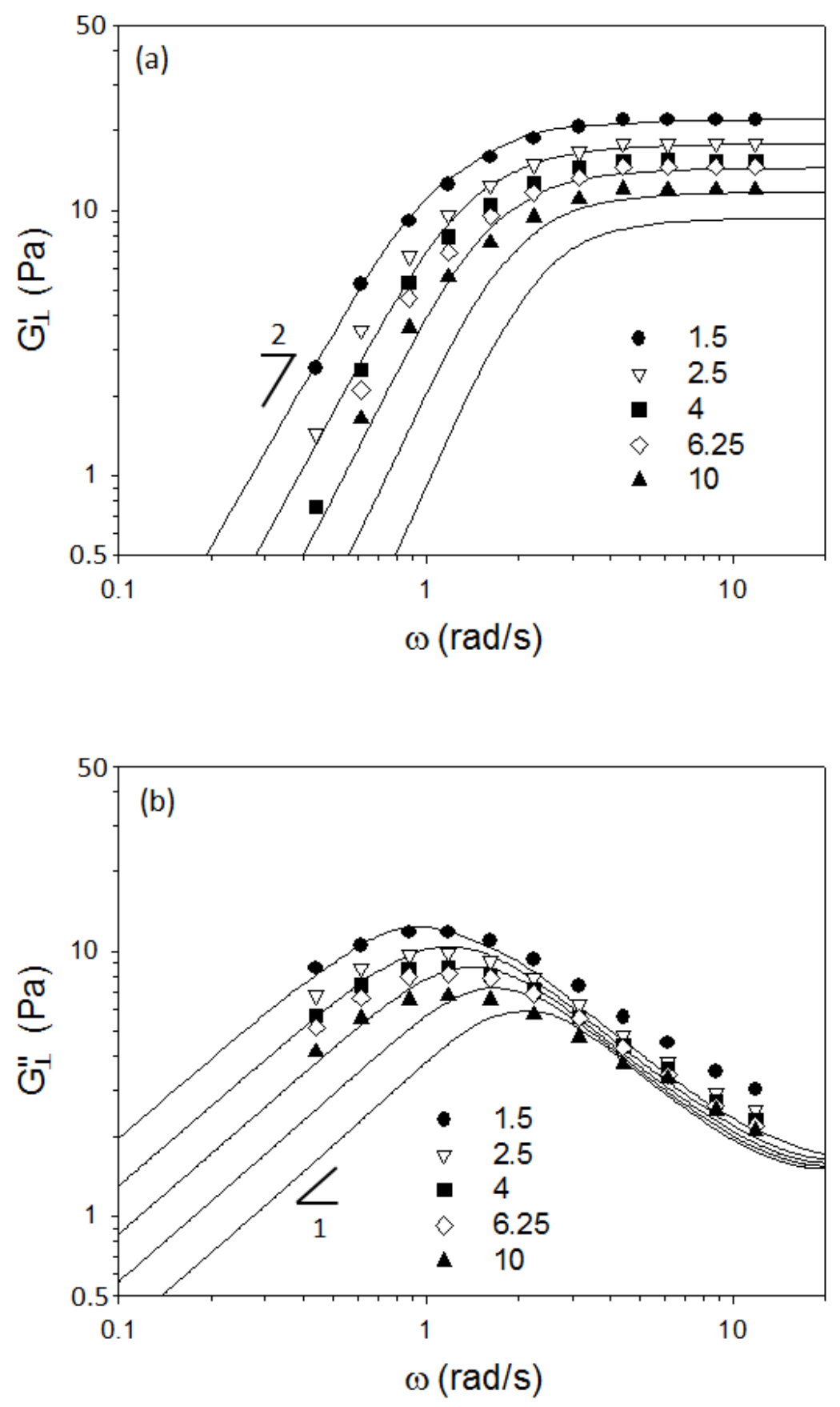

Fig. 7 Orthogonal superposition moduli, (a) $G_{\perp}^{\prime}$ and (b) $G_{\perp}^{\prime \prime}$ at the shear banding regime: experimental (symbols), and calculated values (lines, increasing shear rate from left to right) from the Giesekus model, Eqs. (6) and (7) with model parameters same as used in Fig. 5. 

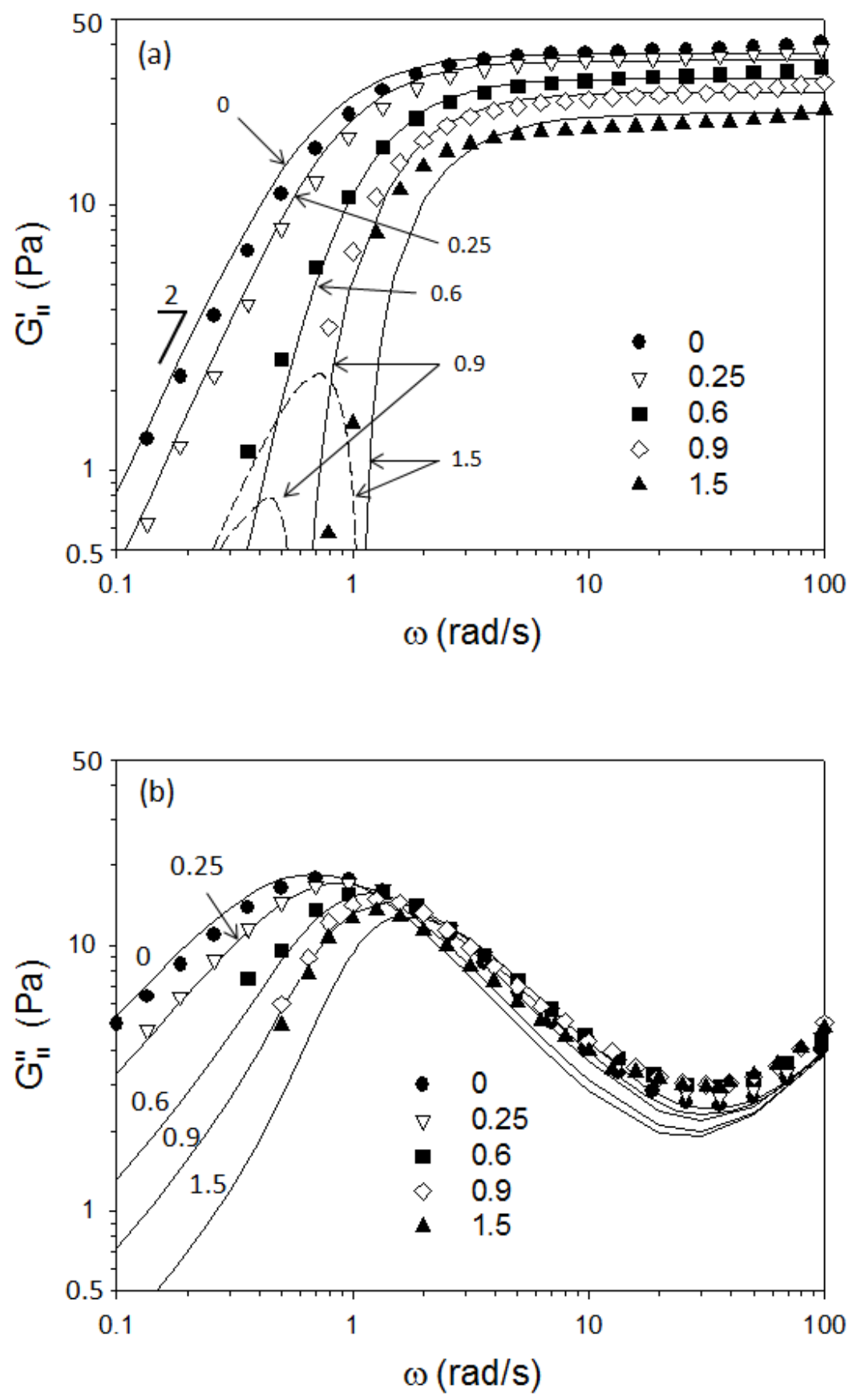

Fig. 8 Parallel superposition moduli $G_{\square}^{\prime}$ and $G_{\square}^{\prime \prime}$ : experimental values (symbols) and calculated values from the Giesekus model (lines) of Eqs. (10) and (11) with the same model parameters as used in Fig. 5. The dashed line in (a) represents absolute values of the negative $G_{\square}^{\prime}$. 

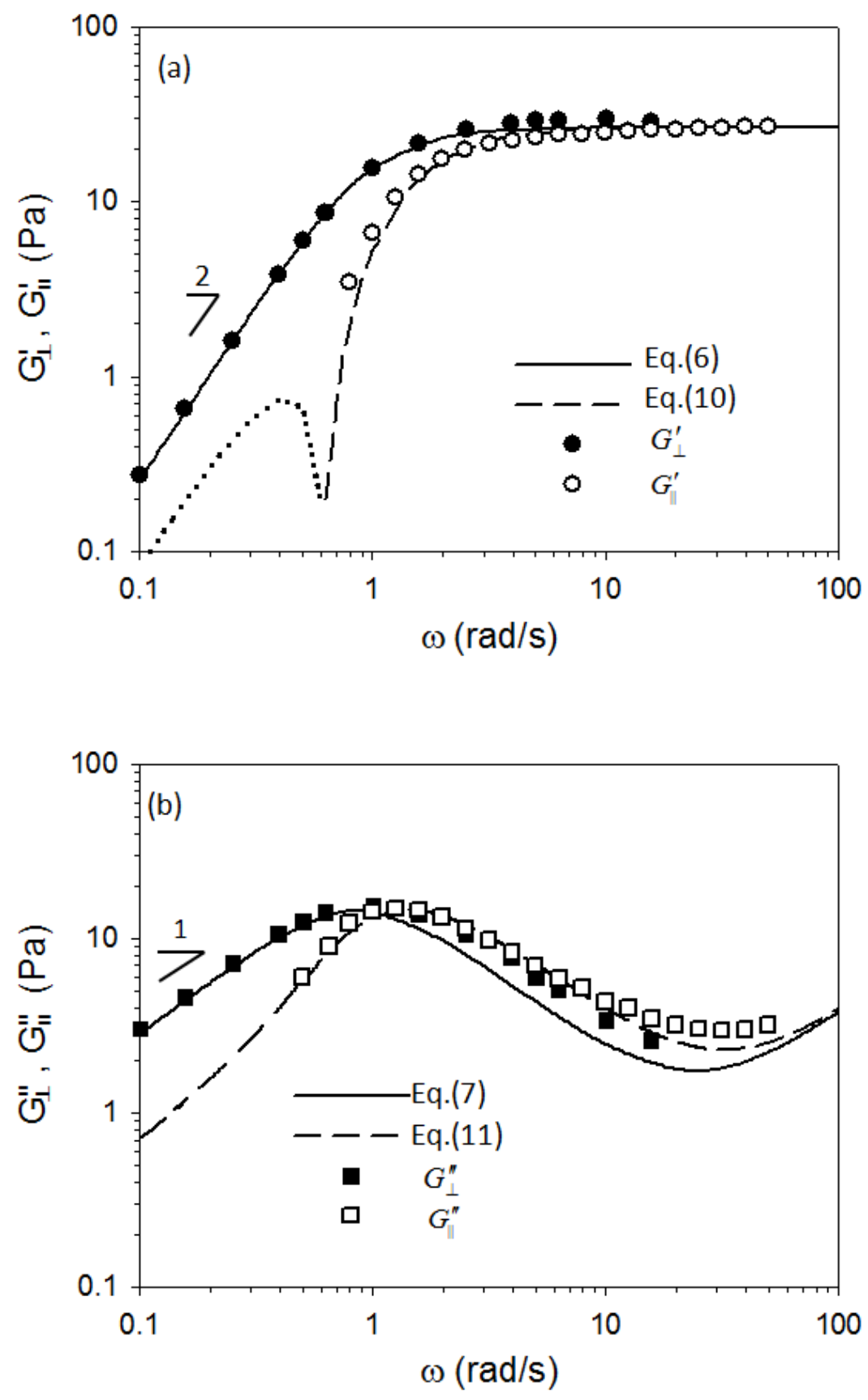

Fig. 9 Parallel and orthogonal moduli at $\dot{\gamma}=0.9 \mathrm{~s}^{-1}$ : experimental values (symbols) and calculated values from the Giesekus model (lines). Dotted lines in (a) represent absolute values of negative $G_{\square}^{\prime}$. 

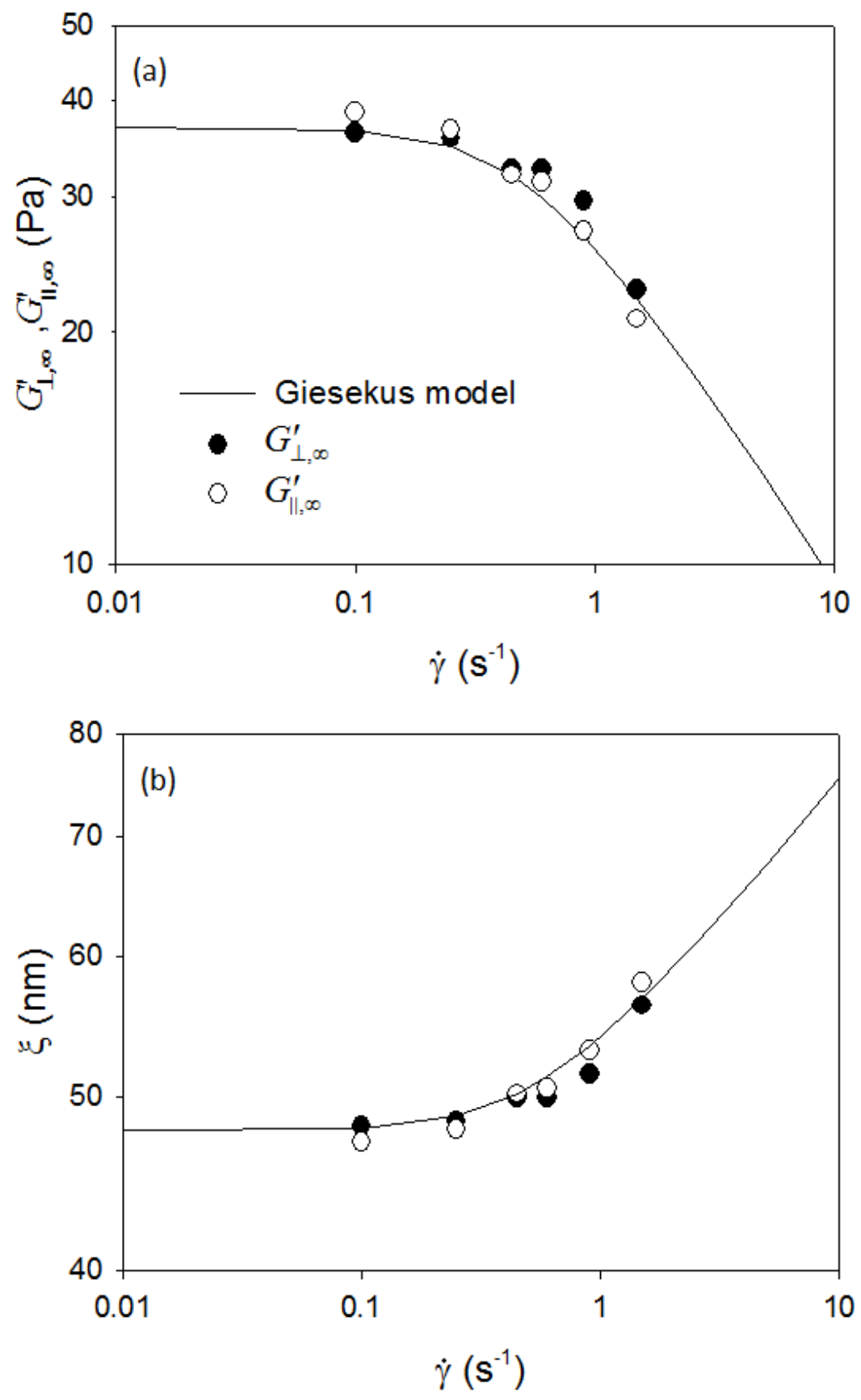

Fig. 10 (a) High frequency value of the superposition moduli as a function of the macroscopic shear rate for parallel and orthogonal superposition and as predicted by the Giesekus model (b) Mesh size calculated from the high frequency moduli. 


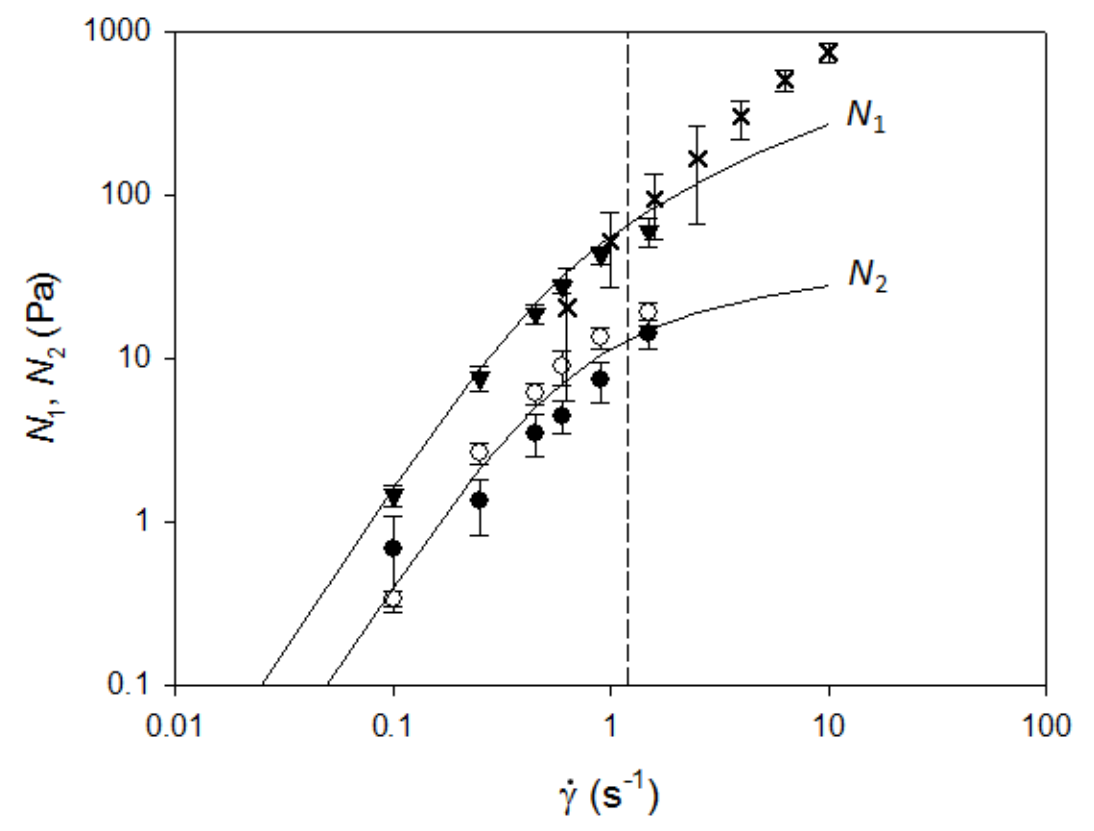

Fig. 11 The first $\left(N_{1}\right)$ and second $\left(N_{2}\right)$ normal stress differences. $\odot$ and $\bullet$ are $N_{2}$ calculated from $G_{\square, \infty}^{\prime}$ and $G_{\perp, \infty}^{\prime}$, respectively using Eq. (14); $\boldsymbol{\nabla}$ is $N_{1}$ calculated from $N_{2}$ using Eq. (25); $\times$ is experimental values of $N_{1}$; the solid lines are $N_{2}$ and $N_{1}$ calculated from the Giesekus model, Eqs. (24) and (25), respectively. The shear banding regime is distinguished from the shear thinning regime by the vertical dashed line. 


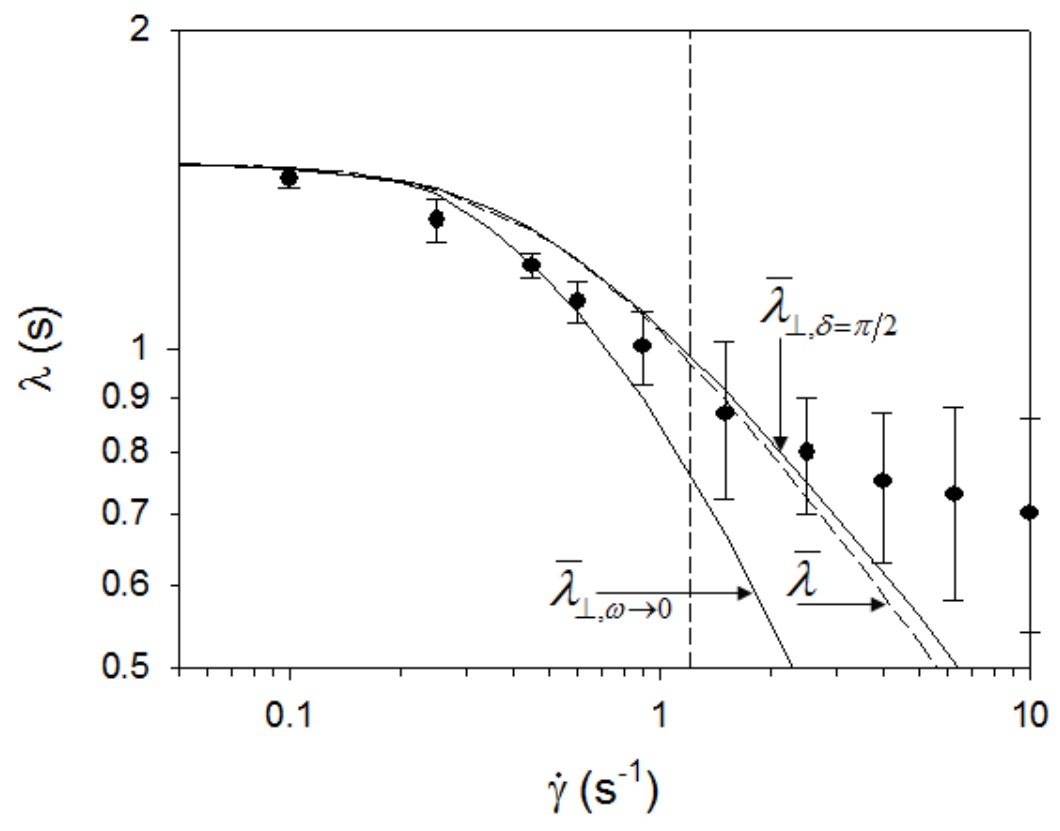

Fig. 12 Comparison of different relaxation times. Solid lines are $\lambda_{\perp, \omega \rightarrow 0}$ and $\lambda_{\perp, \delta=\pi / 2}$, respectively, as obtained from predictions of the orthogonal moduli using the Giesekus model. Dashed lines is $\bar{\lambda}$, obtained from steady shear predictions of the Giesekus model, Eq. (27). - is an experimental values of $\lambda_{\perp, \delta=\pi / 2}$, obtained from orthogonal superposition measurements. The vertical dashed line indicates the onset of the shear banding regime. 


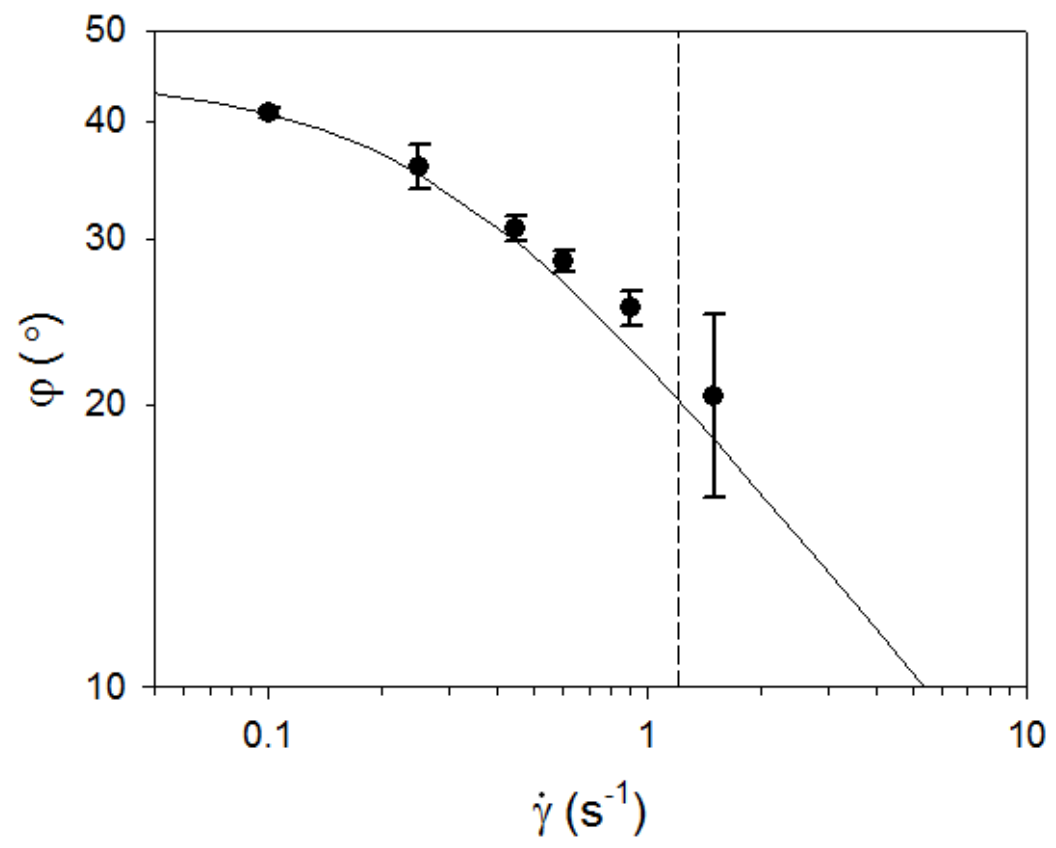

Fig. 13 Orientation angle calculated from Eq. (16) with $\bar{\lambda}_{\perp, \delta=\pi / 2}$ of WLM (symbol) and Giesekus model (solid line) as displayed in Fig 12. The vertical dashed line indicates the onset of the shear banding regime. 


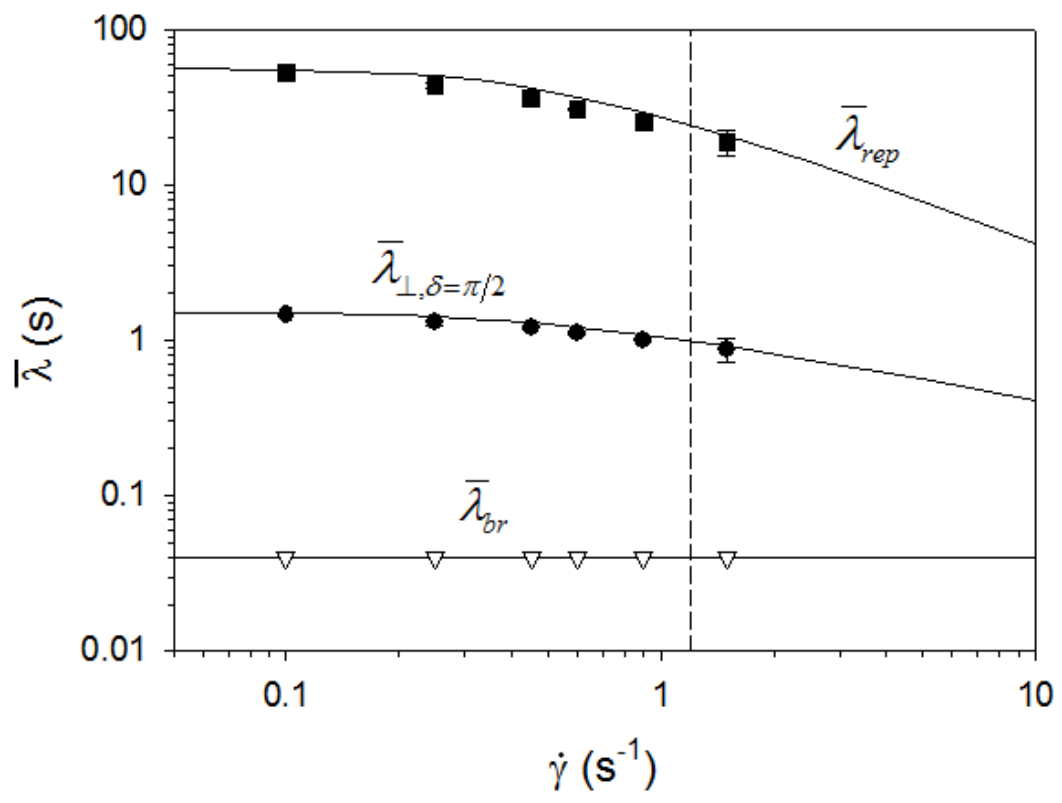

Fig. 14 Reptation time $\bar{\lambda}_{\text {rep }}$ calculated from Eq. (17), using the breakup time $\bar{\lambda}_{b r}$, obtained from the high frequency minimum of the parallel loss moduli in Fig. 8 and the crossover relaxation time $\bar{\lambda}_{\perp, \delta=\pi / 2}$, obtained from WLM (symbols) and the Giesekus model (solid lines). The vertical dashed line indicates the onset of the shear banding regime. 